\title{
Quantum corrections to the ground state of a trapped Bose-Einstein condensate
}

\author{
Eric Braaten \\ Department of Physics, The Ohio State University, Columbus, Ohio 43210 \\ Agustin Nieto \\ Theory Division, CERN, CH-1211 Geneva 23, Switzerland
}

(Received 21 July 1997)

\begin{abstract}
In the mean-field approximation, the number density $\rho(\mathbf{r})$ for the ground state of a Bose-Einstein condensate trapped by an external potential $V(\mathbf{r})$ satisfies a classical field equation called the Gross-Pitaevskii equation. We show that quantum corrections to $\rho$ are dominated by quantum fluctuations with wavelengths of order $1 / \sqrt{\rho a}$, where $a$ is the $S$-wave scattering length. By expanding the equations for the Hartree-Fock approximation to second order in the gradient expansion, we derive local correction terms to the Gross-Pitaevskii equation for $\rho$ that take into account the dominant effects of quantum fluctuations. We also show that the gradient expansions for the density and for the condensate break down at fourth order and at second order, respectively. [S0163-1829(97)07445-6]
\end{abstract}

\section{INTRODUCTION}

The successful achievement of Bose-Einstein condensation of atomic gases in magnetic traps ${ }^{1-3}$ has created an explosion of interest in interacting Bose gases. The condensates in existing magnetic traps are sufficiently dilute that the mean-field approximation gives a satisfactory description of present experimental measurements. However, accurate theoretical predictions require that quantum fluctuations around the mean field also be taken into account. The relative magnitude of these corrections grows as the square root of the number density of the atoms. They will therefore become more important as higher trap densities are achieved and as the precision of experimental measurements improves.

One of the basic observables of a Bose-Einstein condensate trapped in an external potential $V(\mathbf{r})$ is the numberdensity profile $\rho(\mathbf{r})$ of the ground state. In the mean-field approximation, $\rho(\mathbf{r})$ satisfies the time-independent GrossPitaevskii equation

$$
\left(\frac{\hbar^{2}}{2 m} \nabla^{2}+\mu-V(\mathbf{r})\right) \sqrt{\rho}(\mathbf{r})-\frac{4 \pi \hbar^{2} a}{m} \rho \sqrt{\rho}(\mathbf{r})=0,
$$

where $a$ is the $S$-wave scattering length of the atoms. The chemical potential $\mu$ must be tuned so that $\int d^{3} r \rho=N$, where $N$ is the number of atoms in the trap. The density profile of a trapped Bose-Einstein condensate has been studied extensively using Eq. (1). The solutions to this equation have been calculated using numerical methods ${ }^{4,5}$ and variational methods. ${ }^{6-9}$ The solutions have also been studied analytically in the Thomas-Fermi limit, in which the gradient term in Eq. (1) is neglected. ${ }^{6}$ The corrections due to the breakdown of this approximation near the edge of the condensate have also been studied. ${ }^{10,11}$

There are corrections to the mean-field approximation from quantum fluctuations around the mean field. In a dilute homogeneous Bose gas, the relative magnitude of the contributions of quantum fluctuations to thermodynamic quantities is characterized by the dimensionless quantity $\sqrt{\rho a^{3}}$. For condensates in existing magnetic traps, the peak value of $\sqrt{\rho a^{3}}$ is small, but not negligible. Since there are some observables that are more sensitive than the density to the effects of quantum fluctuations, it is important to be able to calculate the effects of quantum fluctuations quantitatively.

In this paper, we calculate the effects of quantum fluctuations on the density profile for a Bose-Einstein condensate in a trapping potential. The expansion parameter that characterizes the relative magnitude of these effects is $\sqrt{\rho a^{3}}$, where $\rho$ is the local number density. We point out that the quantum corrections are dominated by quantum fluctuations with wavelengths on the order of $1 / \sqrt{\rho a}$. The leading effects of these short-distance quantum fluctuations can be calculated using the gradient expansion. By carrying out a selfconsistent one-loop calculation through second order in the gradient expansion, we determine the correction terms that must be added to the Gross-Pitaevskii equation (1) for $\rho$ to take into account the effects of quantum fluctuations:

$$
\begin{aligned}
0= & \left(\frac{\hbar^{2}}{2 m} \nabla^{2}+\mu-V(\mathbf{r})\right) \sqrt{\rho}(\mathbf{r})-\frac{4 \pi \hbar^{2} a}{m} \rho \sqrt{\rho}(\mathbf{r}) \\
& -\frac{128 \sqrt{\pi} \hbar^{2} a^{5 / 2}}{3 m} \rho^{2}(\mathbf{r})-\frac{17 \hbar^{2} a^{3 / 2}}{18 \sqrt{\pi} m}\left[2 \sqrt{\rho} \nabla^{2} \sqrt{\rho}(\mathbf{r})\right. \\
& \left.+(\boldsymbol{\nabla} \sqrt{\rho})^{2}(\mathbf{r})\right] .
\end{aligned}
$$

Our method involves a combination of the Hartree-Fock approach $^{12}$ and the Thomas-Fermi approach. ${ }^{13,14}$ In the Hartree-Fock approximation, which involves the selfconsistent treatment of one-loop quantum corrections, the equation for the density is an integral equation. We obtain the local differential equation (2) by applying a gradient expansion to the integral equation, which corresponds to expanding around the Thomas-Fermi limit.

In the mean-field approximation, the number density is related to the condensate $\langle\psi\rangle$ by $\rho=|\langle\psi\rangle|^{2}$. We find that the gradient expansion for the quantum corrections to this relation breaks down at second order. Thus the effects of quan- 
tum fluctuations on the condensate cannot be summarized by a local differential equation as in Eq. (2). This suggests that it may not be straightforward to generalize the Eq. (2) to the case of a Bose-Einstein condensate containing a vortex. In the presence of a vortex, it is $\langle\psi\rangle$ rather than $\sqrt{\rho}$ that in the mean-field approximation satisfies the time-independent Gross-Pitaevskii equation.

We begin in Sec. II by formulating the problem of BoseEinstein condensation in a trapping potential as a problem in quantum field theory. In Sec. III, we develop a perturbation expansion for calculating the effects of quantum fluctuations around an arbitrary background field. In Sec. IV, we calculate the one-loop quantum corrections to the density profile and the condensate profile. We show that the ultraviolet divergences that arise in the calculation can be removed by the same renormalizations of the action and the number density that are required in the absence of the potential. We find that the number density can be expanded to second order in the gradient of the mean field, while the gradient expansion for the condensate breaks down at that order. In Sec. V, we calculate the self-consistent one-loop quantum corrections to the density profile to second order in the gradient expansion and show that they are given by Eq. (2). We repeat the calculation in Sec. VI using an alternative parametrization for the quantum field and demonstrate that the final result is independent of the parametrization. We also use this parametrization to show that the gradient expansion for the density breaks down at fourth order. Finally, in Sec. VII, we examine the implications of our results for Bose-Einstein condensation in present magnetic traps. Details of the calculations of Feynman diagrams are included in several appendixes.

\section{QUANTUM FIELD THEORY FORMULATION}

Consider a large number $N$ of identical bosonic atoms trapped in an external potential $V(\mathbf{r})$ at zero temperature. If the momenta of the atoms are sufficiently low, their de Broglie wavelengths are much smaller than the range of the interatomic potential, which is comparable in magnitude to the Bohr radius. In this case, the scattering of two atoms will be dominated by $S$-wave scattering and can be described by a single number, the $S$-wave scattering length $a$. Our problem is to determine the number-density profile $\rho(\mathbf{r})$ of the atoms. We begin by formulating this many-body quantum mechanics problem as a problem in quantum field theory.

A convenient way to describe a system containing any number $N$ of atoms is in terms of a quantum field $\psi(\mathbf{r}, t)$ that annihilates an atom. If the atom is a boson, the field satisfies the equal-time commutation relations

$$
\begin{gathered}
{\left[\psi(\mathbf{r}, t), \psi\left(\mathbf{r}^{\prime}, t\right)\right]=0,} \\
{\left[\psi(\mathbf{r}, t), \psi^{\dagger}\left(\mathbf{r}^{\prime}, t\right)\right]=\delta\left(\mathbf{r}-\mathbf{r}^{\prime}\right) .}
\end{gathered}
$$

The time evolution of the field is given by the equation

$$
i \hbar \frac{\partial}{\partial t} \psi=\left[-\frac{\hbar^{2}}{2 m} \nabla^{2}+V(\mathbf{r})\right] \psi+\frac{g+\delta g}{2} \psi^{\dagger} \psi \psi
$$

where the coupling constant $g$ is related to the $S$-wave scattering length $a$ by

$$
g=\frac{8 \pi \hbar^{2} a}{m} .
$$

The parameter $\delta g$ in Eq. (5) is a counterterm associated with the renormalization of $g$. It is necessary to impose an ultraviolet cutoff $\Lambda_{\mathrm{UV}}$ on the wave numbers of virtual particles in order to avoid ultraviolet divergences due to quantum fluctuations of the field. Renormalization of a quantum field theory is the tuning of its parameters so that physical quantities are independent of the ultraviolet cutoff. All the dependence of first-order quantum corrections on $\Lambda_{\mathrm{UV}}$ can be removed by adjusting $\delta g$ in Eq. (5) as a function of $\Lambda_{\mathrm{UV}}$.

The number operator, which counts the number of atoms, is

$$
\hat{N}=\int d^{3} r \psi^{\dagger} \psi(\mathbf{r}, t) .
$$

That this is a number operator follows from the commutation relations (3) and (4), which imply that $\psi^{\dagger}$ and $\psi$ act as raising and lowering operators for $\hat{N}$. Equation (5) implies that $\hat{N}$ is independent of time, so the number of atoms is conserved. Equation (5) can also be expressed in the form

$$
i \hbar \frac{\partial}{\partial t} \psi=-[\hat{H}, \psi]
$$

where the Hamiltonian operator $\hat{H}$ is

$$
\hat{H}=\int d^{3} r\left(\psi^{\dagger}\left[-\frac{\hbar^{2}}{2 m} \nabla^{2}+V(\mathbf{r})\right] \psi+\frac{g+\delta g}{4} \psi^{\dagger} \psi^{\dagger} \psi \psi\right) .
$$

The Hamiltonian $\hat{H}$ measures the energy of the atoms.

The vacuum $|0\rangle$, defined by $\psi(\mathbf{r}, t)|0\rangle=0$, represents the state containing zero atoms. One can show that a Schrödinger wave function for $N$ atoms can be represented as a matrix element of an operator between a state with $\hat{N}=N$ and the vacuum. The simplest case is a state $|\phi\rangle$ containing one atom, which satisfies $\hat{N}|\phi\rangle=|\phi\rangle$. Since the last term in Eq. (5) annihilates the single-particle state $|\phi\rangle$, the matrix element $\langle 0|\psi(\mathbf{r}, t)| \phi\rangle$ satisfies the Schrödinger equation

$$
\left[i \hbar \frac{\partial}{\partial t}+\frac{\hbar^{2}}{2 m} \nabla^{2}-V(\mathbf{r})\right]\langle 0|\psi(\mathbf{r}, t)| \phi\rangle=0 .
$$

Thus $\langle 0|\psi(\mathbf{r}, t)| \phi\rangle$ can be interpreted as the Schrödinger wave function for an atom in the potential $V(\mathbf{r})$.

The next simplest case is a state $|\phi\rangle$ containing two atoms, which satisfies $\hat{N}|\phi\rangle=2|\phi\rangle$. It is straightforward to show using Eq. (5) that the matrix element $\left\langle 0\left|\psi\left(\mathbf{r}_{1}, t\right) \psi\left(\mathbf{r}_{2}, t\right)\right| \phi\right\rangle$ satisfies the Schrödinger equation for two particles in the external potential $V(\mathbf{r})$ interacting through a two-body potential proportional to $\delta^{3}\left(\mathbf{r}_{1}-\mathbf{r}_{2}\right)$. In the absence of the potential $V(\mathbf{r})$, one can calculate the amplitude for the scattering of two atoms exactly. ${ }^{15}$ The scattering amplitude $f(\theta)$ is independent of the scattering angle $\theta$, so it describes $S$-wave scattering. If the total energy of the two atoms in the center-of-momentum frame is $E=2\left(p^{2} / 2 m\right)$, the scattering amplitude is 


$$
f=-\frac{1}{4 \pi}\left[\frac{2 \hbar^{2}}{m(g+\delta g)}+\int \frac{d^{3} k}{(2 \pi)^{3}} \frac{1}{k^{2}-m E / \hbar^{2}-i \epsilon}\right]^{-1} .
$$

The integral over the wave vector $\mathbf{k}$ is ultraviolet divergent. A particularly convenient regularization of the integral is to introduce an ultraviolet cutoff $\Lambda_{\mathrm{UV}}$ through the following prescription:

$$
\begin{aligned}
\int \frac{d^{3} k}{(2 \pi)^{3}} \frac{1}{k^{2}-m E / \hbar^{2}-i \epsilon} \\
\equiv \frac{1}{2 \pi^{2}} \int_{0}^{\infty} k^{2} d k\left(\frac{1}{k^{2}-m E / \hbar^{2}-i \epsilon}-\frac{1}{k^{2}}\right) \\
\quad+\frac{1}{2 \pi^{2}} \int_{0}^{\Lambda_{\mathrm{UV}}} k^{2} d k \frac{1}{k^{2}} .
\end{aligned}
$$

The scattering amplitude then becomes

$$
f=-\frac{1}{4 \pi}\left[\frac{2 \hbar^{2}}{m(g+\delta g)}+\frac{1}{2 \pi^{2}} \Lambda_{\mathrm{UV}}+i \frac{\sqrt{m E}}{4 \pi \hbar}\right]^{-1} .
$$

The dependence on the ultraviolet cutoff can be completely canceled by choosing the bare coupling constant $g+\delta g$ to be

$$
g+\delta g=\frac{g}{1-m g \Lambda_{\mathrm{UV}} /(2 \pi \hbar)^{2}} .
$$

The scattering amplitude (13) then reduces to

$$
f=-\frac{a}{1+i a \sqrt{m E} / \hbar} .
$$

This confirms the identification of $a$ as the $S$-wave scattering length. The scattering of atoms is correctly reproduced by the pointlike interaction term in Eq. (5) as long as the energy of the atoms is sufficiently low that Eq. (13) is a good approximation to the scattering amplitude. Note that the energy dependence in Eq. (15) is that required by the optical theorem.

It is sometimes stated that a $\delta$-function potential in three dimensions is trivial in the sense that it gives no scattering. A more accurate statement is that there is no scattering if we take the ultraviolet cutoff to infinity with the strength of the potential held fixed. This is evident from Eq. (13), which shows that $f \rightarrow 0$ if we take $\Lambda_{\mathrm{UV}} \rightarrow \infty$ with $g+\delta g$ fixed. However, if we allow the strength of the potential to vary with $\Lambda_{\mathrm{UV}}$ in accordance with Eq. (14), we obtain the nontrivial scattering amplitude (15).

From the expression (13) for the scattering amplitude, one can infer an upper limit on the ultraviolet cutoff that must be satisfied in order for perturbative calculations to be accurate. The expansion for $f$ in powers of $g$, including the first quantum correction, which is proportional to $g^{2}$, is

$$
f=-\frac{m g}{8 \pi \hbar^{2}}\left[1+\left(\frac{m g \Lambda_{\mathrm{UV}}}{(2 \pi \hbar)^{2}}-\delta g+i \frac{m g \sqrt{m E}}{8 \pi \hbar^{3}}\right)+\cdots\right] .
$$

If the ultraviolet cutoff $\Lambda_{\mathrm{UV}}$ is too large, there is a delicate cancellation between the term proportional to $\Lambda_{\mathrm{UV}}$ in Eq. (16), which comes from an integral over $k$, and the counterterm $\delta g$. Since a perturbative calculation is necessarily approximate, the cancellation can lead to large errors. Such a large cancellation can be avoided if the term proportional to $\Lambda_{\mathrm{UV}}$ in Eq. (16) is much less than 1. This sets an upper bound on the ultraviolet cutoff:

$$
\Lambda_{\mathrm{UV}} \ll \frac{(2 \pi \hbar)^{2}}{m g}=\frac{\pi}{2 a} .
$$

If this upper bound on $\Lambda_{\mathrm{UV}}$ is not satisfied, then in order to obtain an accurate calculation, it is necessary to use a nonperturbative calculational method that sums up all orders in $g$.

A state $|\phi\rangle$ containing three atoms satisfies $\hat{N}|\phi\rangle=3|\phi\rangle$. In the absence of the potential $V(\mathbf{r})$, one can calculate the amplitude for $3 \rightarrow 3$ scattering as an expansion in powers of $g$. The leading contribution is proportional to $g^{2}$ and comes from two successive $2 \rightarrow 2$ scatterings. Higher-order terms in $g$ represent quantum corrections. The dependence of the first quantum correction on the ultraviolet cutoff $\Lambda_{\mathrm{UV}}$ is canceled by the counterterm $\delta g$ in Eq. (5). However, the second quantum correction, which is proportional to $g^{4}$, has a logarithmic ultraviolet divergence that is not canceled. ${ }^{16}$ Thus corrections to physical quantities from second order in the quantum fluctuations depend on the ultraviolet cutoff. One can eliminate the dependence on $\Lambda_{\mathrm{UV}}$ from second-order quantum corrections by adding to Eq. (5) the term $\left(g_{3}+\delta g_{3}\right) \psi^{\dagger} \psi^{\dagger} \psi \psi \psi / 12$. The logarithmic ultraviolet divergence is canceled by choosing the counterterm to be

$$
\delta g_{3}=\frac{3(4 \pi-3 \sqrt{3})}{32 \pi^{3}} m^{3} g^{4} \log \frac{\Lambda_{\mathrm{UV}}}{\kappa},
$$

where the value of $\kappa$, which was introduced to make the argument of the logarithm dimensionless, depends on the precise definition of $g_{3}$. The parameter $g_{3}$ represents a pointlike contribution to the $3 \rightarrow 3$ scattering amplitude. Thus the $S$-wave scattering length $a$ does not contain enough information about the low-energy scattering of atoms to calculate second-order quantum fluctuations. It is also necessary to know the $3 \rightarrow 3$ coupling constant $g_{3}$. In this paper we will avoid this complication by calculating only to first order in the quantum corrections.

A state $|\phi\rangle$ containing $N$ atoms satisfies $\hat{N}|\phi\rangle=N|\phi\rangle$. In the presence of the potential $V(\mathbf{r})$, the ground state in the $\hat{N}=N$ sector, which we denote by $\left|\Omega_{N}\right\rangle$, is the state that minimizes $\langle\phi|\hat{H}| \phi\rangle$ subject to the constraint $\hat{N}|\phi\rangle=N|\phi\rangle$. The desired number-density profile is

$$
\rho(\mathbf{r})=\left\langle\Omega_{N}\left|\psi^{\dagger} \psi(\mathbf{r})\right| \Omega_{N}\right\rangle .
$$

If $N$ is large, we expect $\rho(\mathbf{r})$ to be insensitive to changes in $N$ that are small compared to $N$. This suggests that we can relax the constraint on the particle number and replace the state $\left|\Omega_{N}\right\rangle$ in Eq. (19) by the state that minimizes $\langle\phi|\hat{H}| \phi\rangle$ subject to the weaker constraint $\langle\phi|\hat{N}| \phi\rangle=N$. If the rootmean-square fluctuations of $\hat{N}$ in that state are small compared to $N$, the expectation value of $\psi^{\dagger} \psi(\mathbf{r})$ in that state 
should give a good approximation to Eq. (19). But that state is precisely the ground state $\left|\Omega_{\mu}\right\rangle$ of the quantum field theory whose Hamiltonian is $\hat{H}-\mu \hat{N}$, where the value of the chemical potential $\mu$ is such that

$$
\left\langle\Omega_{\mu}|\hat{N}| \Omega_{\mu}\right\rangle=N .
$$

Thus, if $N$ is sufficiently large, the density profile can be approximated by the ground-state expectation value of the operator $\psi^{\dagger} \psi(\mathbf{r})$ in the state $\left|\Omega_{\mu}\right\rangle$.

We have now formulated the problem of calculating the density profile as a quantum field theory problem. The field theory is summarized by the action

$$
\begin{aligned}
S[\psi]= & \int d t \int d^{3} r\left\{\psi^{\dagger}\left[i \hbar \frac{\partial}{\partial t}+\frac{\hbar^{2}}{2 m} \nabla^{2}+(\mu+\delta \mu)-V(\mathbf{r})\right] \psi\right. \\
& \left.-\frac{g+\delta g}{4}\left(\psi^{\dagger} \psi\right)^{2}\right\} .
\end{aligned}
$$

The counterterms $\delta \mu$ and $\delta g$ are needed to cancel ultraviolet divergences associated with quantum fluctuations of the field. The counterterm $\delta \mu$ would also have been required in Eq. (5) if the interaction term $\psi^{\dagger} \psi \psi$ had not been normal ordered. A different operator-ordering prescription for $\psi^{\dagger} \psi \psi$ corresponds to adding a term proportional to $\left[\psi, \psi^{\dagger}\right] \psi=\delta^{3}(0) \psi$. The extra term can be canceled by also adding to Eq. (5) a term $-\delta \mu \psi$, with $\delta \mu$ proportional to the ultraviolet divergent constant $\delta^{3}(0)$. While the normalordered prescription is convenient when considering the scattering of atoms in the vacuum, it is awkward for carrying out perturbative calculations in the Bose-condensed state. Rather than specifying an operator-ordering prescription explicitly, it is more convenient to simply use the counterterm $\delta \mu$ to cancel any ultraviolet divergences that might be generated by operator ordering.

The local number-density operator $\psi^{\dagger} \psi(\mathbf{r})$ is also usually defined to be normal ordered. A different operator-ordering prescription corresponds to adding a term proportional to $\left[\psi, \psi^{\dagger}\right]=\delta^{3}(0)$. The extra term can be canceled by subtracting an ultraviolet divergent constant $\delta \rho$ proportional to $\delta^{3}(0)$. The number-density profile is the expectation value of the number density operator in the ground state of the field theory:

$$
\rho(\mathbf{r})=\left\langle\psi^{\dagger} \psi(\mathbf{r})\right\rangle-\delta \rho .
$$

Here and below we use angular brackets to denote the expectation value in the ground state $\left|\Omega_{\mu}\right\rangle$. Rather than specifying an operator-ordering prescription for $\psi^{\dagger} \psi$ explicitly, it is more convenient to simply use the counterterm $\delta \rho$ to cancel any ultraviolet divergences in the number density that are generated by operator ordering. The chemical potential $\mu$ in Eq. (21) must be adjusted so that the integral of the local number density is equal to the number of atoms:

$$
\int d^{3} r \rho(\mathbf{r})=N .
$$

Thus our problem reduces to calculating the ground-state expectation value (22) for the quantum field theory defined by Eq. (21). Another important observable is the condensate $\langle\psi(\mathbf{r})\rangle$, which is the ground-state expectation value of the field. A nonzero value of the condensate indicates the spontaneous breaking of the phase symmetry $\psi \rightarrow e^{i \alpha} \psi$ of the action (21).

The ultraviolet divergences that are canceled by the counterterm $\delta g$ arise from treating the interaction between atoms as pointlike down to arbitrarily short distances. The divergences could be avoided by replacing the pointlike $\left(\psi^{\dagger} \psi\right)^{2}$ interaction term in the action (21) by an interaction through a two-body potential $v\left(\mathbf{r}_{1}-\mathbf{r}_{2}\right)$. A physically realistic twobody potential would have a range comparable to the size of an atom and its shape would have to be adjusted so that it gives the correct $S$-wave scattering length $a$. It would be rather inefficient to calculate the effects of interactions using a physically realistic two-body potential. The reason is that physical quantities depend on the two-body potential in a very simple way. Almost all of the dependence enters through the $S$-wave scattering length $a$. Thus we can obtain the same result for physical quantities by using any simple two-body potential whose inverse range $\Lambda_{\mathrm{UV}}$ is much larger than the momenta of the atoms and whose strength $g+\delta g$ is tuned to give the correct scattering length. The tuning of $\delta g$ makes low-energy $2 \rightarrow 2$ scattering insensitive to the behavior of $v\left(\mathbf{r}_{1}-\mathbf{r}_{2}\right)$ at short distances comparable to $1 / \Lambda_{\mathrm{UV}}$. This tuning is also sufficient to make the first-order quantum corrections to other low-energy observables insensitive to the short-distance behavior of the two-body potential. At higher order in the quantum corrections, there are additional parameters that must be tuned. For example, at second order in the quantum corrections, it is necessary to also tune the strength $g_{3}+\delta g_{3}$ of a three-body potential. However, as long as one considers only first-order quantum corrections, all lowenergy observables can be calculated in terms of the single parameter $a$.

At short distances, a slowly varying external potential $V(\mathbf{r})$ in Eq. (21) is equivalent to a shift in the chemical potential $\mu$. It has no effect on the scattering of atoms and therefore does not change the value of the counterterm $\delta g$ that is required to tune the scattering length to the value $a$. The counterterms $\delta \mu$ and $\delta \rho$ associated with operator ordering are also independent of $V(\mathbf{r})$. Thus the renormalizations required to remove ultraviolet divergences in the case of a Bose gas in a trapping potential are identical to those required for a homogeneous Bose gas.

An alternative way to deal with ultraviolet divergences is to replace the interatomic potential $(g+\delta g) \delta^{3}(\mathbf{r}) / 2$ by a pseudopotential $g \delta^{3}(\mathbf{r})(\partial / \partial r) r / 2 .{ }^{17}$ Ultraviolet divergences can be avoided by evaluating the partial derivative in the pseudopotential at the appropriate stage of the calculation. We find it simpler to introduce an ultraviolet cutoff and use the renormalization machinery of quantum field theory to remove the dependence on the cutoff.

Most previous work on the density profile has been carried out within the mean-field approximation. In this approximation, quantum fluctuations are neglected. The counterterms $\delta \mu, \delta g$, and $\delta \rho$, which cancel ultraviolet divergences associated with those quantum fluctuations, can all be set to zero. The field $\psi$ satisfies the time-dependent Gross-Pitaevskii equation, which is the classical field equation for the action (21): 


$$
i \hbar \frac{\partial}{\partial t} \psi+\left(\frac{\hbar^{2}}{2 m} \nabla^{2}+\mu-V(\mathbf{r})\right) \psi-\frac{g}{2}\left(\psi^{\dagger} \psi\right) \psi=0 .
$$

The ground state $\left|\Omega_{\mu}\right\rangle$ corresponds to a time-independent solution $\phi_{0}(\mathbf{r})$ that can be chosen to be real valued. The mean field therefore satisfies

$$
\left(\frac{\hbar^{2}}{2 m} \nabla^{2}+\mu-V(\mathbf{r})\right) \phi_{0}(\mathbf{r})-\frac{g}{2} \phi_{0}^{3}(\mathbf{r})=0 .
$$

The number density (22) reduces to

$$
\rho(\mathbf{r})=\phi_{0}^{2}(\mathbf{r})
$$

Thus the density profile in the mean-field approximation satisfies the Gross-Pitaevskii equation (1). The condensate in the mean-field approximation is

$$
\langle\psi(\mathbf{r})\rangle=\phi_{0}(\mathbf{r}),
$$

and it therefore satisfies $\langle\psi\rangle=\sqrt{\rho}$.

The density profile is modified by quantum corrections. The corrections can be obtained by expanding the quantum field around the mean field $\phi_{0}(\mathbf{r})$, which satisfies Eq. (25):

$$
\psi(\mathbf{r}, t)=\phi_{0}(\mathbf{r})+\widetilde{\psi}(\mathbf{r}, t) .
$$

The expression (22) for the number density becomes

$$
\rho(\mathbf{r})=\phi_{0}^{2}(\mathbf{r})+2 \phi_{0}(\mathbf{r}) \operatorname{Re}\langle\widetilde{\psi}(\mathbf{r})\rangle+\left\langle\widetilde{\psi}^{\dagger} \widetilde{\psi}(\mathbf{r})\right\rangle-\delta \rho .
$$

The condensate differs from $\sqrt{\rho}$ because of the effects of quantum fluctuations:

$$
\langle\psi(\mathbf{r})\rangle=\phi_{0}(\mathbf{r})+\langle\widetilde{\psi}(\mathbf{r})\rangle .
$$

Having formulated our problem in terms of quantum field theory, there are quantum fluctuations on all length scales ranging from $L$, the length scale associated with variations in $\rho(\mathbf{r})$, down to the inverse of the ultraviolet cutoff $\Lambda_{\mathrm{UV}}$. The quantum fluctuations with length scales of order $L$ depend in detail on the shape of the potential $V(\mathbf{r})$. For quantum fluctuations with length scales much smaller than $L$, the effects of variations in $V(\mathbf{r})$ are negligible. The short-distance quantum fluctuations therefore behave locally like those of a homogeneous Bose gas with chemical potential $\mu-V(\mathbf{r})$. We will show that these fluctuations give the dominant corrections to the density profile.

The one-loop quantum corrections to the number density can be obtained by keeping the terms in the action that are quadratic in the fluctuating fields $\widetilde{\psi}(\mathbf{r}, t)$. If these fields are expanded in terms of normal modes, the corrections (29) to $\rho(\mathbf{r})$ can be expressed as a sum over the normal modes. The contribution of an individual normal mode to the number density scales like $1 / L^{3}$. This is negligible compared to the contribution from the mean field, which scales like $N / L^{3}$. A significant contribution can only arise from summing over a large number of normal modes. Normal modes with very short wavelengths approach a continuum and can be labeled by the wave vector $\mathbf{k}$. The contribution to the density from such modes scales like $\int d^{3} k$. The integral is ultraviolet divergent. The ultraviolet divergence is proportional to $\Lambda_{\mathrm{UV}}^{3}$ and is canceled by the counterterm $\delta \rho$ in Eq. (29). After renormalization, modes with $k$ comparable to $\Lambda_{\mathrm{UV}}$ do not contribute to $\rho(\mathbf{r})$. Since the density of modes grows rapidly with $k$, the dominant quantum corrections to $\rho(\mathbf{r})$ come from the largest values of $k$ whose effects are not removed by renormalization. To understand the scale of $k$ that dominates, it is useful to recall some simple facts about the homogeneous Bose gas.

The properties of a homogeneous Bose gas with positive scattering length $a$ and low number density $\rho$ are well understood. The dimensionless quantity $\sqrt{\rho a^{3}}$ serves as an expansion parameter for the low-density expansion. For example, the ground-state energy density, including the first quantum correction, is

$$
\mathcal{E}=\frac{2 \pi \hbar^{2} a \rho^{2}}{m}\left(1+\frac{128}{15 \sqrt{\pi}} \sqrt{\rho a^{3}}\right) .
$$

The coefficient of $\sqrt{\rho a^{3}}$ in the quantum correction term was first obtained by Lee and Yang. ${ }^{18}$ The quasiparticle excitations of the system are Bogoliubov modes, which are plane waves with the dispersion relation

$$
\epsilon(k)=\frac{\hbar^{2} k \sqrt{k^{2}+\Lambda^{2}}}{2 m} .
$$

This dispersion relation changes from linear in $k$ to quadratic at a scale $\Lambda$ given by

$$
\Lambda=\sqrt{16 \pi a \rho} .
$$

This is the scale of the wave number $k$ that dominates the quantum corrections to the energy density. The one-loop quantum correction is the sum over normal modes of the zero-point energies $\hbar \omega / 2$, where $\omega$ is the angular frequency of the normal mode. The contribution from large $k$ behaves like $\int d^{3} k \epsilon(k) / 2$, where $\epsilon(k)$ is the Bogoliubov dispersion relation given in Eq. (32). This integral is ultraviolet divergent, with the leading divergence proportional to $\hbar^{2} \Lambda_{\mathrm{UV}}^{5} / m$. This leading divergence and the subleading divergences can all be removed by renormalization. After renormalization, the integral is dominated by the scale $\Lambda$ given in Eq. (33) and it therefore scales like $\hbar^{2} \Lambda^{5} / m \sim \hbar^{2} \rho^{5 / 2} a^{5 / 2} / m$. This estimate agrees with the explicit result given in Eq. (31).

Generalizing to the case of a nonhomogeneous Bose gas, we can anticipate that the quantum corrections to the density profile $\rho(\mathbf{r})$ will be dominated locally by modes with wave number $k$ on the order of $\sqrt{16 \pi a \rho(\mathbf{r})}$. The contributions from much shorter wavelengths are removed by renormalization. The contributions from much longer wavelengths are suppressed by phase space. These modes can be approximated by a continuum as long as the corresponding wavelengths are much shorter than the scale $L$ for significant variations in $\rho(\mathbf{r})$ :

$$
\sqrt{16 \pi a \rho(\mathbf{r})} \gg \frac{2 \pi}{L} .
$$

If this lower bound on the density is satisfied, then the methods of continuum quantum field theory can be used to calculate the dominant quantum corrections. The condition (34) is also necessary in order to calculate quantum corrections using a gradient expansion, which is an expansion in $1 / \Lambda L$. There is an upper bound on $\rho(\mathbf{r})$ that must be satisfied in 
order to allow perturbative calculations in the quantum field theory with the pointlike interaction in Eq. (21). This condition is that the scale $\Lambda$ must be much less than the maximum ultraviolet cutoff given in Eq. (17):

$$
\sqrt{16 \pi a \rho(\mathbf{r})} \ll \frac{\pi}{2 a}
$$

If this upper bound is not satisfied, then nonperturbative methods must be used to calculate the effects of quantum corrections. The condition (35) is also necessary in order for the effects of quantum fluctuations to be small enough to be treated as perturbative corrections to the mean-field approximation. For example, in the case of a homogeneous Bose gas, the condition that the quantum correction to the energy density, which is given by the second term in Eq. (31), is small compared to the mean-field contribution is essentially identical to Eq. (35). In our analysis of quantum corrections to the density profile, we will assume that the number density is in the range specified by the inequalities (34) and (35).

\section{PERTURBATIVE FRAMEWORK}

In this section we present a general framework for carrying out perturbative calculations of the effects of quantum fluctuations around an arbitrary time-independent background $v(\mathbf{r})$. In Sec. IV, we will set $v$ equal to the mean field $\phi_{0}$ and use this framework to calculate one-loop corrections to the condensate and density profiles. In Sec. V, we set $v$ equal to the condensate $\langle\psi\rangle$ and determine the self-consistent one-loop corrections to the equation for the density profile.

It is convenient to parametrize the quantum field $\psi(\mathbf{r}, t)$ in terms of two real-valued quantum fields $\xi$ and $\eta$ that describe quantum fluctuations around an arbitrary timeindependent background $v(\mathbf{r})$ :

$$
\psi(\mathbf{r}, t)=v(\mathbf{r})+\frac{\xi(\mathbf{r}, t)+i \eta(\mathbf{r}, t)}{\sqrt{2}}
$$

We will refer to this as the Cartesian parametrization of the quantum field. An alternative field parametrization is considered in Sec. VI. If the phase of $\psi$ is chosen so that $v$ is real valued, the condensate profile is

$$
\langle\psi(\mathbf{r})\rangle=v(\mathbf{r})+\frac{1}{\sqrt{2}}\langle\xi(\mathbf{r})\rangle .
$$

The number density is

$$
\rho(\mathbf{r})=v^{2}(\mathbf{r})+\sqrt{2} v(\mathbf{r})\langle\xi(\mathbf{r})\rangle+\frac{1}{2}\left\langle\xi^{2}(\mathbf{r})\right\rangle+\frac{1}{2}\left\langle\eta^{2}(\mathbf{r})\right\rangle-\delta \rho .
$$

Inserting the field parametrization (36) into the action (21) and expanding in powers of the quantum fields $\xi$ and $\eta$, it becomes

$$
\begin{aligned}
S[\psi]= & S[v]+\int d t \int d^{3} r\left\{\sqrt{2} T \xi+\frac{1}{2}(\eta \dot{\xi}-\xi \dot{\eta})\right. \\
& +\frac{1}{4 m} \xi\left(\nabla^{2}-\Lambda^{2}+X\right) \xi+\frac{1}{4 m} \eta\left(\nabla^{2}+Y\right) \eta \\
& \left.+\frac{1}{\sqrt{2}} Z \xi\left(\xi^{2}+\eta^{2}\right)-\frac{g+\delta g}{16}\left(\xi^{2}+\eta^{2}\right)^{2}\right\},
\end{aligned}
$$

where $\dot{f} \equiv(\partial / \partial t) f$ and $T, X, Y$, and $Z$ are external sources that depend on $v$ :

$$
\begin{gathered}
T(\mathbf{r})=\left[(\mu+\delta \mu)-V(\mathbf{r})-\frac{g+\delta g}{2} v^{2}(\mathbf{r})\right] v(\mathbf{r})+\frac{1}{2 m} \nabla^{2} v(\mathbf{r}) \\
X(\mathbf{r})=\Lambda^{2}+2 m\left[(\mu+\delta \mu)-V(\mathbf{r})-\frac{3(g+\delta g)}{2} v^{2}(\mathbf{r})\right] \\
Y(\mathbf{r})=2 m\left[(\mu+\delta \mu)-V(\mathbf{r})-\frac{g+\delta g}{2} v^{2}(\mathbf{r})\right] \\
Z(\mathbf{r})=-\frac{g+\delta g}{2} v(\mathbf{r}) .
\end{gathered}
$$

We have set $\hbar=1$ in the action. Dimensional analysis can be used to reinsert the factors of $\hbar$ at the end of the calculation. The parameter $\Lambda$ appears both in the source $X$ and explicitly in the $\xi^{2}$ term in the action and cancels between them. The arbitrariness of this parameter can be exploited to simplify calculations.

To organize the quantum corrections into a loop expansion, we separate the terms in the action that depend on $\xi$ and $\eta$ into a free part and an interaction part:

$$
S[\psi]=S[v]+S_{\text {free }}[\xi, \eta]+S_{\text {int }}[v, \xi, \eta] .
$$

The free part of the action is

$$
\begin{aligned}
S_{\text {free }}[\xi, \eta]= & \int d t \int d^{3} r\left\{\frac{1}{2}(\eta \dot{\xi}-\xi \dot{\eta})+\frac{1}{4 m} \xi\left(\nabla^{2}-\Lambda^{2}\right) \xi\right. \\
& \left.+\frac{1}{4 m} \eta \nabla^{2} \eta\right\} .
\end{aligned}
$$

This action describes Bogoliubov modes with the dispersion relation (32), where $\Lambda$ is now an adjustable parameter. The Fourier transform of the propagator for the fields $\xi$ and $\eta$ is a $2 \times 2$ matrix

$$
\begin{aligned}
& \left(\begin{array}{cc}
D^{\xi \xi}(\mathbf{k}, \omega) & D^{\xi \eta}(\mathbf{k}, \omega) \\
D^{\eta \xi}(\mathbf{k}, \omega) & D^{\eta \eta}(\mathbf{k}, \omega)
\end{array}\right) \\
& \quad=\frac{1}{\omega^{2}-\epsilon^{2}(k)+i 0^{+}}\left(\begin{array}{cc}
k^{2} / 2 m & -i \omega \\
i \omega & 2 m \epsilon^{2}(k) / k^{2}
\end{array}\right)
\end{aligned}
$$

where $\mathbf{k}$ is the wave vector and $\omega$ is the frequency. The diagonal elements of the propagator matrix (46) are represented by solid lines for $\xi$ and dashed lines for $\eta$, as illustrated in Figs. 1(a) and 1(b). The off-diagonal elements are 
(a)

(b)

(c)

FIG. 1. Diagrammatic representation of the components of the $2 \times 2$ propagator matrix: (a) the diagonal propagator for $\xi$, (b) the diagonal propagator for $\eta$, and (c) the off-diagonal propagator for $\xi$ and $\eta$.

represented by a line that is half solid and half dotted, as in Fig. 1(c). All the remaining terms in the action (39) are treated as interactions:

$$
\begin{aligned}
S_{\text {int }}[v, \xi, \eta]= & \int d t \int d^{3} r\left\{\sqrt{2} T \xi+\frac{1}{4 m} X \xi^{2}+\frac{1}{4 m} Y \eta^{2}\right. \\
& \left.+\frac{1}{\sqrt{2}} Z \xi\left(\xi^{2}+\eta^{2}\right)-\frac{g+\delta g}{16}\left(\xi^{2}+\eta^{2}\right)^{2}\right\} .
\end{aligned}
$$

They include interactions with the external sources $T, X, Y$, and $Z$ as well as the four-point coupling $g+\delta g$. The sources are represented by dots labeled by the appropriate letter, as illustrated in Fig. 2. The four-point couplings are represented by points that connect four lines.

It is possible to diagonalize the propagator matrix (46) by applying a Bogoliubov transformation to the fields $\xi$ and $\eta$. However, such a transformation makes the interaction terms in the action significantly more complicated and increases the number of diagrams that contribute to most quantities. For explicit calculations, it is more economical to minimize

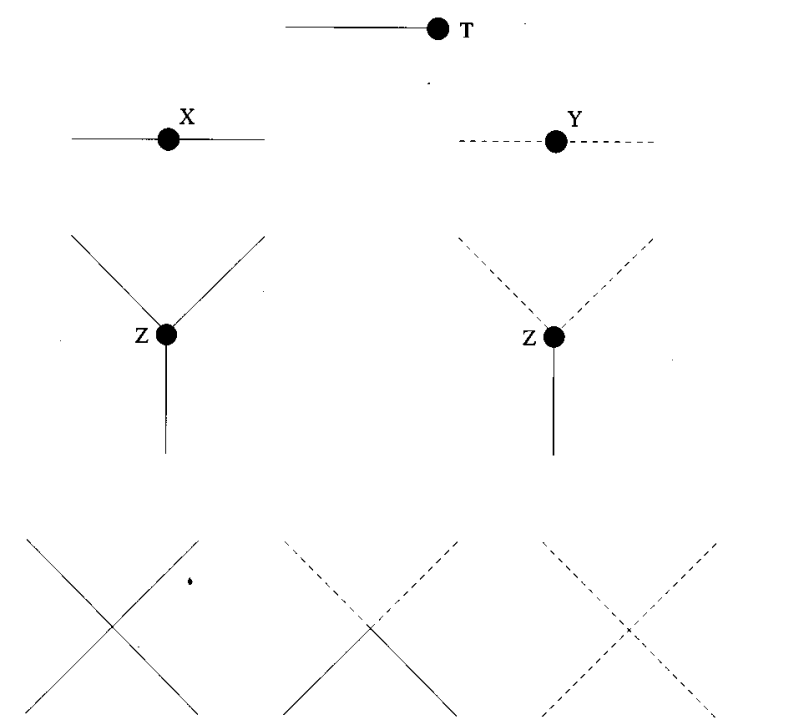

FIG. 2. Diagrammatic representation of the interaction vertices associated with the sources $T, X, Y$, and $Z$ and the four-point couplings.

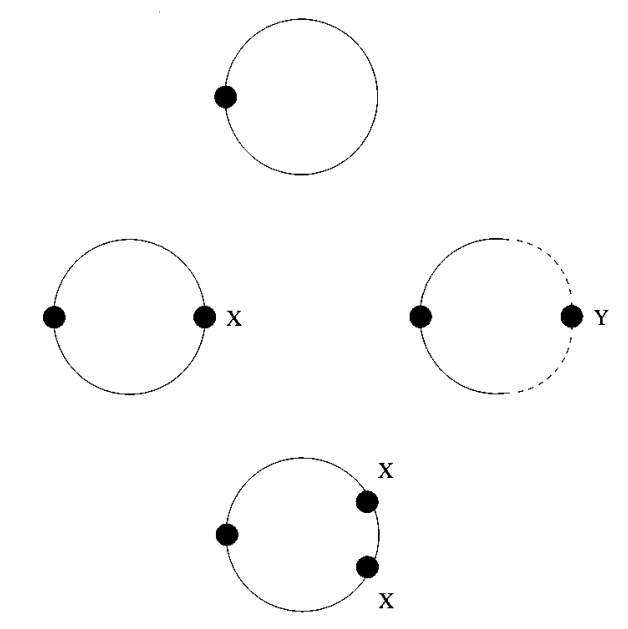

FIG. 3. One-loop Feynman diagrams that contribute to $\left\langle\xi^{2}\right\rangle$.

the number of diagrams. We therefore prefer to use a propagator matrix with off-diagonal elements.

In the case of a homogeneous Bose gas, the trapping potential $V$ is zero and we can choose the background field $v$ to be a constant, independent of $\mathbf{r}$. If we choose $v^{2}=2(\mu+\delta \mu) /(g+\delta g)$ and $\Lambda^{2}=2 m(g+\delta g) v^{2}$, then the sources $T, X$, and $Y$ in Eq. (47) vanish and the interactions reduce to three-point couplings and four-point couplings. Such a perturbative framework has been used recently to reproduce the classic one-loop corrections to the thermodynamic properties of a homogeneous Bose gas. ${ }^{19}$

The leading quantum corrections to the ground-state expectation values in Eqs. (37) and (38) are given by one-loop Feynman diagrams. Examples of one-loop diagrams that contribute to $\left\langle\xi^{2}\right\rangle$ and $\left\langle\eta^{2}\right\rangle$ are shown in Figs. 3 and 4, respectively. The dot on the left-hand side of each diagram represents the operator $\xi^{2}$, which creates two solid lines, or the operator $\eta^{2}$, which creates two dashed lines. The lines form a loop that can include any number of insertions of the sources $X$ and $Y$. It is convenient to introduce the notation \langle\rangle$_{X Y}$ for the expectation value of an operator in the presence of the sources $X$ and $Y$, but with no other self-interactions for the quantum fields. The sum of all one-loop diagrams for $\left\langle\xi^{2}\right\rangle$ and $\left\langle\eta^{2}\right\rangle$ can then be represented as

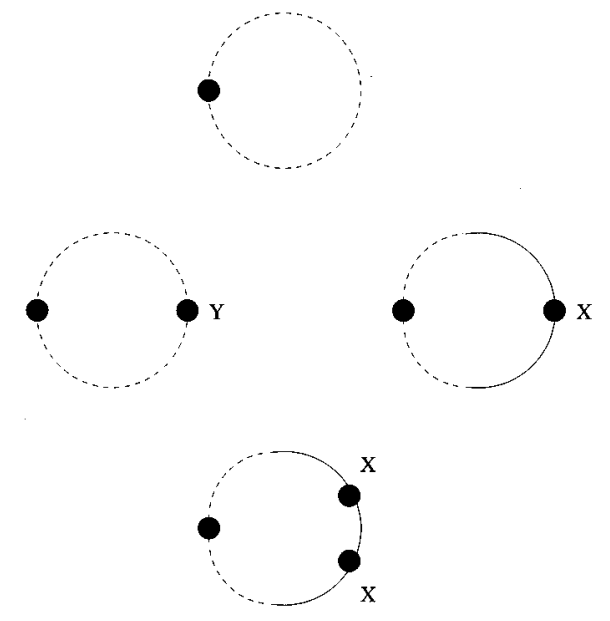

FIG. 4. One-loop Feynman diagrams that contribute to $\left\langle\eta^{2}\right\rangle$. 


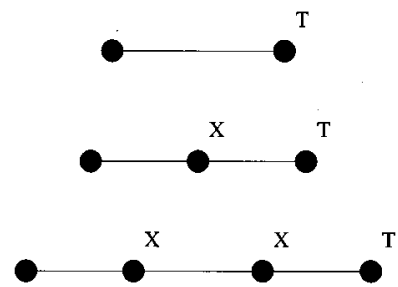

FIG. 5. Feynman diagrams contributing to $\langle\xi\rangle$ that involve the source $T$.

$$
\begin{gathered}
\left.\left\langle\xi^{2}(\mathbf{r})\right\rangle\right|_{1 \text {-loop }}=\left\langle\xi^{2}(\mathbf{r})\right\rangle_{X, Y}, \\
\left.\left\langle\eta^{2}(\mathbf{r})\right\rangle\right|_{1-\text { loop }}=\left\langle\eta^{2}(\mathbf{r})\right\rangle_{X, Y} .
\end{gathered}
$$

The advantage of this notation is that the expectation value $\langle\xi\rangle$ at one-loop order can also be expressed succinctly in terms of $\left\langle\xi^{2}\right\rangle_{X, Y}$ and $\left\langle\eta^{2}\right\rangle_{X, Y}$. Examples of diagrams that contribute to $\langle\xi\rangle$ are shown in Figs. 5-7. The operator $\xi$ creates a single solid line. In the diagrams of Fig. 5, the $\xi$ field propagates to a source $T$, where it is annihilated. In the diagrams of Figs. 6 and 7, it propagates to a source $Z$, which creates a pair of solid lines or dashed lines that form a loop. In all of these diagrams, the $\xi$ propagator and the propagators inside the loop can include any number of insertions of the sources $X$ and $Y$. The sum of all such diagrams can be expressed as
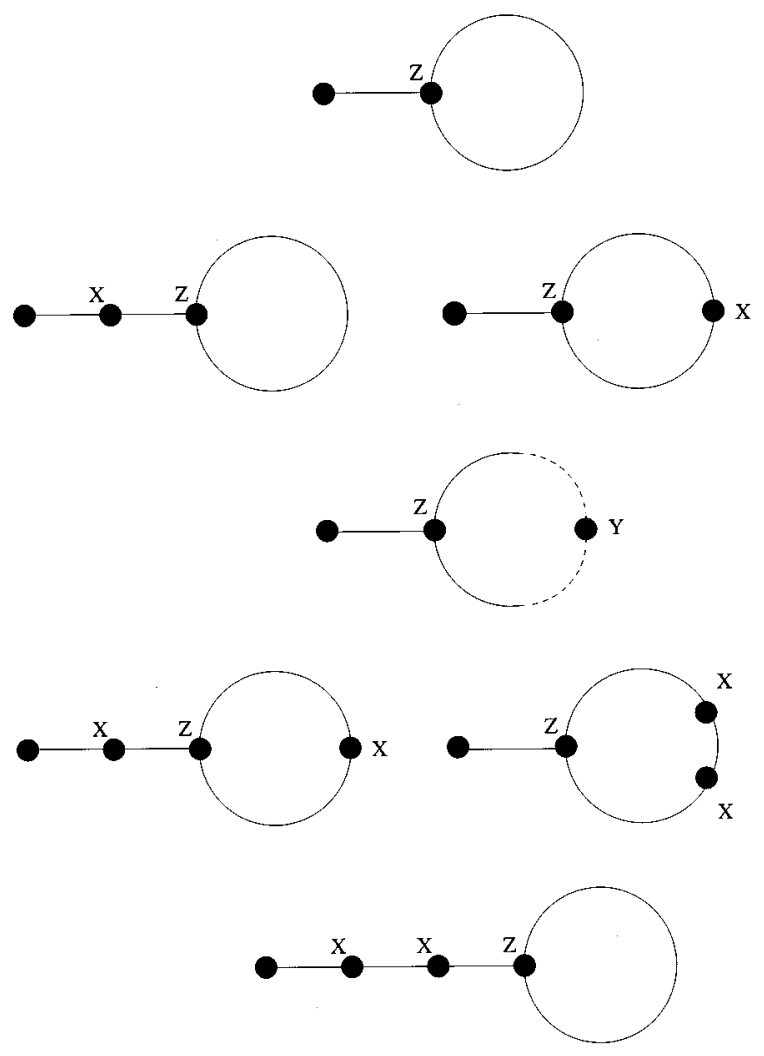

FIG. 6. One-loop Feynman diagrams contributing to $\langle\xi\rangle$ that involve a pair of $\xi$ lines produced by the source $Z$.

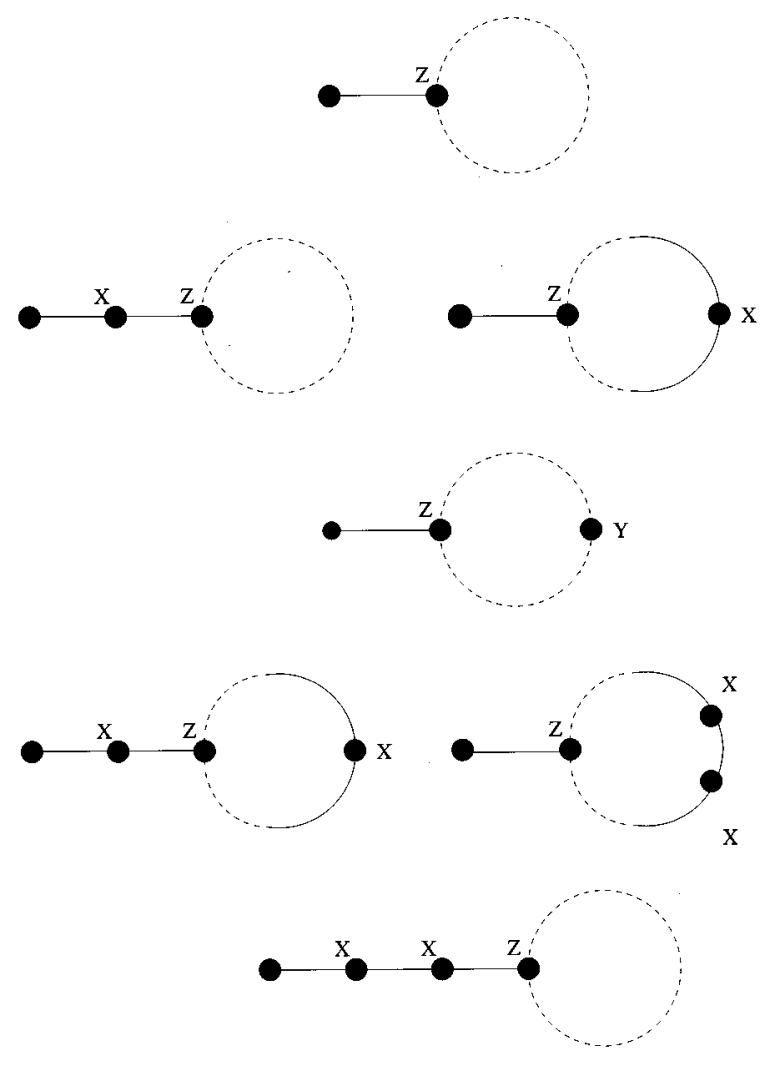

FIG. 7. One-loop Feynman diagrams contributing to $\langle\xi\rangle$ that involve a pair of $\eta$ lines produced by the source $Z$.

$$
\begin{aligned}
\left.\langle\xi(\mathbf{r})\rangle\right|_{1-\text { loop }}= & -\sqrt{2} \int d^{3} r^{\prime}\left(\int d t^{\prime} D_{X, Y}^{\xi \xi}\left(\mathbf{r}, \mathbf{r}^{\prime}, t^{\prime}\right)\right) \\
& \times\left(T\left(\mathbf{r}^{\prime}\right)+\frac{3}{2} Z\left(\mathbf{r}^{\prime}\right)\left\langle\xi^{2}\left(\mathbf{r}^{\prime}\right)\right\rangle_{X, Y}\right. \\
& \left.+\frac{1}{2} Z\left(\mathbf{r}^{\prime}\right)\left\langle\eta^{2}\left(\mathbf{r}^{\prime}\right)\right\rangle_{X, Y}\right),
\end{aligned}
$$

where $D_{X, Y}^{\xi \xi}$ is the diagonal component of the propagator for $\xi$ in the presence of the sources $X$ and $Y$.

The quantities $\left\langle\xi^{2}\right\rangle_{X, Y}$ and $\left\langle\eta^{2}\right\rangle_{X, Y}$ in Eqs. (48)-(50) are functionals of the sources $X$ and $Y$. These functionals include terms of arbitrarily high orders in $X$ and $Y$. They are nonlocal because the loop diagrams involve an integral over the positions of the sources $X$ and $Y$. After renormalization, these integrals are dominated by wavelengths of order $2 \pi / \Lambda$, while the sources vary significantly only over much larger distances of order $L$. It is therefore reasonable to expand the sources $X\left(\mathbf{r}^{\prime}\right)$ and $Y\left(\mathbf{r}^{\prime}\right)$ as Taylor series around the point $\mathbf{r}$. This reduces the expressions for $\left\langle\xi^{2}\right\rangle_{X, Y}$ and $\left\langle\eta^{2}\right\rangle_{X, Y}$ to an infinite sum of local quantities involving $X, Y$, and their derivatives at the point $\mathbf{r}$ :

$$
\begin{aligned}
\left\langle\xi^{2}(\mathbf{r})\right\rangle_{X, Y}= & a_{0}+a_{1} X(\mathbf{r})+a_{2} Y(\mathbf{r})+a_{3} \nabla^{2} X(\mathbf{r})+a_{4} X^{2}(\mathbf{r}) \\
& +a_{5}(\boldsymbol{\nabla} X)^{2}(\mathbf{r})+\cdots \\
\left\langle\eta^{2}(\mathbf{r})\right\rangle_{X, Y}= & b_{0}+b_{1} X(\mathbf{r})+b_{2} Y(\mathbf{r})+b_{3} \nabla^{2} X(\mathbf{r})+b_{4} X^{2}(\mathbf{r}) \\
& +b_{5}(\nabla X)^{2}(\mathbf{r})+\cdots
\end{aligned}
$$


The terms on the right-hand sides of Eqs. (51) and (52) include all possible rotationally invariant combinations of $X$ and $Y$ and their derivatives. The constraint of rotational invariance arises from the rotational invariance of the free action (45). In Eqs. (51) and (52) we have shown explicitly only those terms that will ultimately be needed to calculate the quantum corrections to the density profile.

The coefficients $a_{i}$ and $b_{i}$ in Eq. (51) and (52) can be reduced to integrals over a wave vector $\mathbf{k}$, as illustrated by the explicit calculation of a diagram presented in Appendix B. Having expanded the sources as Taylor series around $\mathbf{r}$, the only scale in the integrand is $\Lambda$. By dimensional analysis, a convergent integral must have the form of the appropriate power of $\Lambda$ multiplied by a numerical coefficient. However, some of the integrals have infrared or ultraviolet divergences and thus require infrared or ultraviolet cutoffs. The ultraviolet divergences either cancel in quantities such as $\rho(\mathbf{r})$ and $\langle\psi(\mathbf{r})\rangle$ or they are removed by renormalization. Infrared divergences reflect a failure of the assumption that the sources can be expanded in a Taylor series inside the loop integral. If these divergences do not cancel, it simply indicates a breakdown of the gradient expansion due to the sensitivity of the quantum corrections to nonlocal effects involving the length scale $L$.

The propagator factor $\int d t^{\prime} D_{X, Y}^{\xi \xi}\left(\mathbf{r}, \mathbf{r}^{\prime}, t^{\prime}\right)$ in Eq. (50) can be expanded in powers of $X$ and its derivatives at the point $\mathbf{r}$. The dependence on the source $Y$ is removed by the integration over $t^{\prime}$, which corresponds to evaluating the Fouriertransformed propagator at $\omega=0$. Since the off-diagonal com-

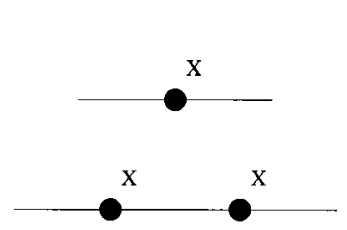

FIG. 8. Feynman diagrams that contribute to the propagator $D_{X, Y}^{\xi \xi}$ at zero frequency.

ponents of the propagator (46) vanish at zero frequency, the source $Y$ does not contribute. Examples of diagrams that contribute to $\int d t^{\prime} D_{X, Y}^{\xi \xi}$ are shown in Fig. 8. The contribution from the first diagram is given by the upper-left component of the propagator matrix in Eq. (46):

$$
\int d t^{\prime} D^{\xi \xi \xi}\left(\mathbf{r}, \mathbf{r}^{\prime}, t^{\prime}\right)=-2 m \int \frac{d^{3} k}{(2 \pi)^{3}} e^{-i \mathbf{k} \cdot\left(\mathbf{r}-\mathbf{r}^{\prime}\right)} \frac{1}{k^{2}+\Lambda^{2}}
$$

The other diagrams in Fig. 8 involve integrals over the positions $\mathbf{r}^{\prime \prime}$ of sources $X\left(\mathbf{r}^{\prime \prime}\right)$. In coordinate space, the propagator factor (53) falls exponentially when $\left|\mathbf{r}-\mathbf{r}^{\prime}\right|$ exceeds $1 / \Lambda$. If we assume that the source $X$ varies significantly only over a much greater length scale $L$, then we can expand $X\left(\mathbf{r}^{\prime \prime}\right)$ as a Taylor series around the point $\mathbf{r}^{\prime \prime}=\mathbf{r}$. The function $\int d t^{\prime} D_{X, Y}^{\xi \xi}\left(\mathbf{r}, \mathbf{r}^{\prime}, t^{\prime}\right)$ can then be expressed in terms of $X(\mathbf{r})$ and its derivatives at the point $\mathbf{r}$. The terms coming from the diagrams in Fig. 8 include

$$
\begin{aligned}
\int d t^{\prime} D_{X, Y}^{\xi \xi \xi}\left(\mathbf{r}, \mathbf{r}^{\prime}, t^{\prime}\right)= & -2 m \int \frac{d^{3} k}{(2 \pi)^{3}} e^{-i \mathbf{k} \cdot\left(\mathbf{r}-\mathbf{r}^{\prime}\right)}\left\{\frac{1}{k^{2}+\Lambda^{2}}+X(\mathbf{r}) \frac{1}{\left(k^{2}+\Lambda^{2}\right)^{2}}-2 i \nabla_{i} X(\mathbf{r}) \frac{k^{i}}{\left(k^{2}+\Lambda^{2}\right)^{3}}\right. \\
& \left.+\nabla_{i} \nabla_{j} X(\mathbf{r})\left[\frac{\delta^{i j}}{\left(k^{2}+\Lambda^{2}\right)^{3}}-4 \frac{k^{i} k^{j}}{\left(k^{2}+\Lambda^{2}\right)^{4}}\right]+2 \nabla_{i} X \nabla_{j} X(\mathbf{r})\left[\frac{\delta^{i j}}{\left(k^{2}+\Lambda^{2}\right)^{4}}-6 \frac{k^{i} k^{j}}{\left(k^{2}+\Lambda^{2}\right)^{5}}\right]+\cdots\right\} .
\end{aligned}
$$

The complete expression involves all possible powers of $X$ and gradients of $X$.

The result (54) can be used to express $\langle\psi(\mathbf{r})\rangle$ and $\rho(\mathbf{r})$ as an expansion in powers of $X$ and $Y$ and their derivatives at the point $(\mathbf{r})$. In the expression for $\langle\xi(\mathbf{r})\rangle$ in Eq. (50), the propagator factor is integrated against a function $f\left(\mathbf{r}^{\prime}\right)$ that depends on the sources $T, Z, X$, and $Y$. The integral can be evaluated by expanding $f\left(\mathbf{r}^{\prime}\right)$ as a Taylor series around the point $\mathbf{r}^{\prime}=\mathbf{r}$. Using the expression (54) for the propagator factor, we can evaluate the integral over $\mathbf{r}^{\prime}$. The resulting expression for the integral includes the terms

$$
\begin{aligned}
& \int d^{3} r^{\prime}\left(\int d t^{\prime} D_{X, Y}^{\xi \xi}\left(\mathbf{r}, \mathbf{r}^{\prime}, t^{\prime}\right)\right) f\left(\mathbf{r}^{\prime}\right) \\
& =-2 m\left\{\left[\frac{1}{\Lambda^{2}}+\frac{1}{\Lambda^{4}} X(\mathbf{r})+\frac{1}{\Lambda^{6}} \nabla^{2} X(\mathbf{r})+\frac{2}{\Lambda^{8}}(\nabla X)^{2}(\mathbf{r})\right]\right.
\end{aligned}
$$

$$
\left.\times f(\mathbf{r})+\frac{2}{\Lambda^{6}} \boldsymbol{\nabla} X(\mathbf{r}) \cdot \boldsymbol{\nabla} f(\mathbf{r})+\frac{1}{\Lambda^{4}} \nabla^{2} f(\mathbf{r})+\cdots\right\} .
$$

Applying this formula to the integral in Eq. (50) and using the expansions (51) and (52) for $\left\langle\xi^{2}\right\rangle$ and $\left\langle\eta^{2}\right\rangle$, we obtain an expansion for $\langle\xi\rangle$ in powers of $X, Y$, and their derivatives. Inserting the expansions for $\langle\xi\rangle,\left\langle\xi^{2}\right\rangle$, and $\left\langle\eta^{2}\right\rangle$ into Eqs. (37) and (38), we obtain expansions for the condensate and the density in powers of $X, Y$, and their derivatives.

\section{ONE-LOOP CALCULATION}

In this section we calculate the one-loop quantum corrections to the density profile $\rho(\mathbf{r})$ and to the condensate profile $\langle\psi(\mathbf{r})\rangle$ to second order in the gradient expansion. The appro priate choice for the background field $v$ is the mean field $\phi_{0}$, which satisfies Eq. (25): 


$$
v(\mathbf{r})=\phi_{0}(\mathbf{r}) .
$$

The quantum fields $\xi$ and $\eta$ in Eq. (36) describe quantum fluctuations around the mean field. The condensate profile (37) reduces to

$$
\langle\psi(\mathbf{r})\rangle=\phi_{0}(\mathbf{r})+\frac{1}{\sqrt{2}}\langle\xi(\mathbf{r})\rangle,
$$

while the number density (38) reduces to

$$
\begin{aligned}
\rho(\mathbf{r})= & \phi_{0}^{2}(\mathbf{r})+\sqrt{2} \phi_{0}(\mathbf{r})\langle\xi(\mathbf{r})\rangle+\frac{1}{2}\left\langle\xi^{2}(\mathbf{r})\right\rangle \\
& +\frac{1}{2}\left\langle\eta^{2}(\mathbf{r})\right\rangle-\delta \rho .
\end{aligned}
$$

The fact that the mean field $\phi_{0}$ satisfies the classical equation (25) can be used to simplify the expressions (40)-(43) for the sources. We can also drop the counterterms $\delta \mu$ and $\delta g$ in the sources $X, Y$, and $Z$. These sources appear only in diagrams that are at least first order in the loop expansion. The counterterms appearing in these sources are therefore needed only to cancel ultraviolet divergences that arise at second order or higher in the loop expansion. Thus the sources can be simplified to

$$
\begin{gathered}
T(\mathbf{r})=\delta \mu \phi_{0}(\mathbf{r})-\frac{\delta g}{2} \phi_{0}^{3}(\mathbf{r}), \\
X(\mathbf{r})=\Lambda^{2}-2 m g \phi_{0}^{2}(\mathbf{r})-\frac{\nabla^{2} \phi_{0}}{\phi_{0}}(\mathbf{r}), \\
Y(\mathbf{r})=-\frac{\nabla^{2} \phi_{0}}{\phi_{0}}(\mathbf{r}), \\
Z(\mathbf{r})=-\frac{g}{2} \phi_{0}(\mathbf{r}) .
\end{gathered}
$$

The expressions (57) and (58) for the condensate and the density are nonlocal functionals of the mean field $\phi_{0}$. If $\langle\psi(\mathbf{r})\rangle$ and $\rho(\mathbf{r})$ are expanded in powers of the sources $X$ and $Y$ and their derivatives at the point $\mathbf{r}$, the expansions include infinitely many terms. They can be reduced to local functionals of $\phi_{0}$ by consistently truncating the expansions. We will reduce Eqs. (57) and (58) to local equations at a specific point $\mathbf{r}_{0}$ by (a) choosing a specific value for the arbitrary parameter $\Lambda$ and (b) truncating the equations at second order in the gradient expansion. Note that the source $Y$ in Eq. (61) is already second order in the gradient expansion. Thus, if we truncate the equations at second order in the gradient expansion, we need only include terms up to first order in $Y$ and we can omit all derivatives of $Y$. We also need only include terms up to first order in $\nabla^{2} X$ and up to second order in $\nabla X$. However, we still must include all possible powers of $X$.

In order to reduce the expansions for $\langle\psi\rangle$ and $\rho$ to a finite number of terms, we will choose $\Lambda$ so that $X\left(\mathbf{r}_{0}\right)$ is second order in the gradient expansion at a specific point $\mathbf{r}_{0}$. If we evaluate $\langle\psi\rangle$ and $\rho$ at the point $\mathbf{r}_{0}$ and then truncate them at second order in gradients of $\phi_{0}$, the resulting expressions for $\left\langle\psi\left(\mathbf{r}_{0}\right)\right\rangle$ and $\rho\left(\mathbf{r}_{0}\right)$ are algebraic functions of $\phi_{0}, \nabla \phi_{0}$, and $\nabla^{2} \phi_{0}$ evaluated at the point $\mathbf{r}_{0}$. Since we could have chosen any particular point for $\mathbf{r}_{0}$, these algebraic relations must hold at any point $\mathbf{r}$. The most convenient choice for $\Lambda$ is the wave number that appears in the Bogoliubov dispersion relation (32) for a homogeneous gas with number density $\phi_{0}^{2}\left(\mathbf{r}_{0}\right)$ :

$$
\Lambda^{2}=2 m g \phi_{0}^{2}\left(\mathbf{r}_{0}\right) \text {. }
$$

The source $X$ and its derivatives at the point $\mathbf{r}_{0}$ then reduce to

$$
\begin{gathered}
X\left(\mathbf{r}_{0}\right)=-\frac{\nabla^{2} \phi_{0}}{\phi_{0}}\left(\mathbf{r}_{\mathbf{0}}\right), \\
\boldsymbol{\nabla} X\left(\mathbf{r}_{0}\right)=-4 m g \phi_{0} \boldsymbol{\nabla} \phi_{0}\left(\mathbf{r}_{0}\right), \\
\nabla^{2} X\left(\mathbf{r}_{0}\right)=-4 m g\left[\phi_{0} \nabla^{2} \phi_{0}+\left(\boldsymbol{\nabla} \phi_{0}\right)^{2}\right]\left(\mathbf{r}_{0}\right) .
\end{gathered}
$$

We proceed to calculate the one-loop correction to the condensate $\langle\psi(\mathbf{r})\rangle$, which is given by Eq. (50). Inserting Eqs. (51) and (52) into Eq. (50) and using Eq. (55) to evaluate the integral over $\mathbf{r}^{\prime}$, we obtain an expansion for $\langle\xi\rangle$ in powers of the sources and their derivatives. Inserting the expressions (59), (61), (62), and (64)-(66) for the sources at the point $\mathbf{r}_{0}$, we obtain

$$
\begin{aligned}
\left\langle\xi\left(\mathbf{r}_{0}\right)\right\rangle= & -\sqrt{2} m g \phi_{0}\left\{\left[\frac{3 a_{0}+b_{0}}{2}-2 \frac{\delta \mu}{g}+\frac{\delta g}{g} \phi_{0}^{2}\right] \frac{1}{\Lambda^{2}}\right. \\
& -\left[\left(3 a_{0}+b_{0}-4 \frac{\delta \mu}{g}\right) \frac{1}{\Lambda^{4}}+\frac{9 a_{1}+3 b_{1}+3 a_{2}+b_{2}}{2} \frac{1}{\Lambda^{2}}+\left(3 a_{3}+b_{3}\right)\right] \frac{\nabla^{2} \phi_{0}}{\phi_{0}} \\
& \left.+\left[\left(3 a_{0}+b_{0}-4 \frac{\delta \mu}{g}\right) \frac{1}{\Lambda^{4}}+\left(3 a_{1}+b_{1}\right) \frac{1}{\Lambda^{2}}-\left(3 a_{3}+b_{3}-12 a_{4}-4 b_{4}\right)+2\left(3 a_{5}+b_{5}\right) \Lambda^{2}\right] \frac{\left(\nabla \phi_{0}\right)^{2}}{\phi_{0}^{2}}\right\} .
\end{aligned}
$$


The coefficients $a_{i}$ and $b_{i}$ are given in Appendix C. The coefficients $a_{0}$ and $b_{0}$ are cubically ultraviolet divergent, while $a_{1}$ and $b_{1}$ are linearly divergent. The divergences are canceled by taking the counterterms $\delta \mu$ and $\delta g$ to have the values

$$
\begin{gathered}
\delta \mu=\frac{1}{12 \pi^{2}} g \Lambda_{\mathrm{UV}}^{3}, \\
\delta g=\frac{1}{4 \pi^{2}}\left(m g \Lambda_{\mathrm{UV}}\right) g .
\end{gathered}
$$

The counterterm $\delta g$ in Eq. (69) agrees with that obtained by expanding Eq. (14) to first order in $m g \Lambda_{\mathrm{Uv}}$. Using the results for $a_{i}$ and $b_{i}$ in Appendix $\mathrm{C}$ and the value of $\Lambda$ given in Eq. (63), the condensate at the point $\mathbf{r}_{0}$ reduces to

$$
\begin{aligned}
\langle\psi\rangle= & \phi_{0}\left\{1-\frac{5}{48 \pi^{2}}(2 m g)^{3 / 2} \phi_{0}\right. \\
& -\frac{1}{16 \pi^{2}} \sqrt{2 m g}\left[\left(\frac{49}{18}-\frac{5}{24} \log \frac{8 m g \phi_{0}^{2}}{\lambda_{\mathrm{IR}}^{2}}\right) \frac{\nabla^{2} \phi_{0}}{\phi_{0}^{2}}\right. \\
& \left.\left.+\left(\frac{29}{9}-\frac{1}{16} \log \frac{8 m g \phi_{0}^{2}}{\lambda_{\mathrm{IR}}^{2}}\right) \frac{\left(\nabla \phi_{0}\right)^{2}}{\phi_{0}^{3}}\right]\right\},
\end{aligned}
$$

where $\lambda_{\mathrm{IR}}$ is an infrared cutoff. The logarithmic infrared divergences arise from the coefficients $b_{2}, b_{3}$, and $b_{5}$. The divergences indicate that the gradient expansion for the condensate breaks down at second order. Thus we can obtain a local expression for the condensate only to leading order in the gradient expansion. Keeping only the first correction term in Eq. (70), the result is

$$
\langle\psi(\mathbf{r})\rangle=\phi_{0}(\mathbf{r})\left[1-\frac{5}{48 \pi^{2}}(2 m g)^{3 / 2} \phi_{0}(\mathbf{r})\right] .
$$

We derived this equation at the point $\mathbf{r}_{0}$ defined by our choice (63) for the arbitrary parameter $\Lambda$. However, our final result for $\left\langle\psi\left(\mathbf{r}_{0}\right)\right\rangle$ is an algebraic expression in terms of $\phi_{0}\left(\mathbf{r}_{0}\right)$. Since we could have chosen any particular point for $\mathbf{r}_{0}$, that algebraic expression must be valid at any point $\mathbf{r}$.

We next calculate the one-loop corrections to the density, which is given by Eq. (58). The expression for $\langle\xi\rangle$ at the point $\mathbf{r}_{0}$ is given by Eq. (67). The corresponding expressions for $\left\langle\xi^{2}\right\rangle$ and $\left\langle\eta^{2}\right\rangle$ are obtained by inserting the expressions (61) and (64)-(66) for the sources at the point $\mathbf{r}_{0}$ into Eqs. (51) and (52). The resulting expression for the density at the point $\mathbf{r}_{0}$ is

$$
\begin{aligned}
\rho\left(\mathbf{r}_{0}\right)= & \phi_{0}^{2}-\left[a_{0}+\delta \rho-2 \frac{\delta \mu}{g}+\frac{\delta g}{g} \phi_{0}^{2}\right] \\
& +\left[\left(3 a_{0}+b_{0}-4 \frac{\delta \mu}{g}\right) \frac{1}{\Lambda^{2}}+\left(4 a_{1}+b_{1}+a_{2}\right)\right. \\
& \left.+2 a_{3} \Lambda^{2}\right] \frac{\nabla^{2} \phi_{0}}{\phi_{0}} \\
& -\left[\left(3 a_{0}+b_{0}-4 \frac{\delta \mu}{g}\right) \frac{1}{\Lambda^{2}}+\left(3 a_{1}+b_{1}\right)\right. \\
& \left.-2\left(a_{3}-6 a_{4}-2 b_{4}\right) \Lambda^{2}+4 a_{5} \Lambda^{4}\right] \frac{\left(\nabla \phi_{0}\right)^{2}}{\phi_{0}^{2}} .
\end{aligned}
$$

After using the expressions (68) and (69) for the counterterms $\delta \mu$ and $\delta g$, the only remaining ultraviolet divergence is a cubic divergence that can be canceled by choosing the density counterterm to be

$$
\delta \rho=\frac{1}{12 \pi^{2}} \Lambda_{\mathrm{UV}}^{3} .
$$

The infrared divergent coefficients $b_{2}, b_{3}$, and $b_{5}$ have canceled in the expression (72) for the number density. Thus the density has a well-defined gradient expansion through second order, in contrast to the condensate. Our final expression for the number density, including one-loop quantum corrections, is

$$
\begin{aligned}
\rho(\mathbf{r})= & \phi_{0}^{2}(\mathbf{r})\left\{1-\frac{1}{6 \pi^{2}}(2 m g)^{3 / 2} \phi_{0}(\mathbf{r})\right. \\
& \left.-\frac{1}{16 \pi^{2}} \sqrt{2 m g}\left[\frac{41}{9} \frac{\nabla^{2} \phi_{0}}{\phi_{0}^{2}}(\mathbf{r})+\frac{113}{18} \frac{\left(\boldsymbol{\nabla} \phi_{0}\right)^{2}}{\phi_{0}^{3}}(\mathbf{r})\right]\right\} .
\end{aligned}
$$

We derived this equation at the specific point $\mathbf{r}_{0}$. However, our final expressions for $\rho\left(\mathbf{r}_{0}\right)$ is an algebraic expression involving $\phi_{0}, \nabla \phi_{0}$, and $\nabla^{2} \phi_{0}$ evaluated at the point $\mathbf{r}_{0}$. Since we could have chosen any particular point for $\mathbf{r}_{0}$, these algebraic relations must hold at any point $\mathbf{r}$.

Combining Eqs. (71) and (74), we obtain a local expression for the condensate in terms of the density that is correct to leading order in the gradient expansion:

$$
\langle\psi(\mathbf{r})\rangle=\sqrt{\rho}(\mathbf{r})\left[1-\frac{1}{48 \pi^{2}}(2 m g)^{3 / 2} \sqrt{\rho}(\mathbf{r})\right] .
$$

This agrees with a result obtained recently by Timmermans, Tommasini, and Huang. ${ }^{14}$

The choice (63) for $\Lambda$ is not unique. Any choice that makes $X\left(\mathbf{r}_{0}\right)$ second order in the gradient expansion will be equally acceptable and must give the same final answer. For example, we could have chosen

$$
\Lambda^{2}=2 m g \phi_{0}^{2}\left(\mathbf{r}_{0}\right)+\frac{\nabla^{2} \phi_{0}}{\phi_{0}}\left(\mathbf{r}_{0}\right) .
$$

In that case, Eq. (64) would be replaced by $X\left(\mathbf{r}_{0}\right)=0$. Following the effects of this change through the calculation, we 
find that the coefficient $4 a_{1}$ of $\nabla^{2} \phi_{0} / \phi_{0}$ in Eq. (72) is replaced by $3 a_{1}$. However, the term $-a_{0}$ in Eq. (72) depends on $\Lambda$, which is given in Eq. (76). When this term is expanded in powers of gradients of $\phi_{0}$, it generates additional terms proportional to $\nabla^{2} \phi_{0} / \phi_{0}$ that precisely cancel the change in Eq. (72). Thus we recover the same final result (74).

Note that the counterterms (68), (69), and (73) do not depend on the potential $V(\mathbf{r})$. Thus the ultraviolet divergences in one-loop diagrams are removed by the same renormalizations that are required for a homogeneous Bose gas.

\section{SELF-CONSISTENT ONE-LOOP CALCULATION}

In this section we present a self-consistent one-loop calculation of the equation for the density profile $\rho(\mathbf{r})$ to second order in the gradient expansion. The calculation involves taking the equations for the density in the Hartree-Fock approximation and expanding them around the Thomas-Fermi limit. The result is the differential equation (2) that generalizes the Gross-Pitaevskii equation for $\rho$ by taking into account the leading effects of quantum fluctuations.

The self-consistent one-loop equations can be expressed as classical field equations for the one-loop effective action. ${ }^{20}$ We describe briefly the diagrammatic representation of these equations. They correspond to summing all connected diagrams with arbitrarily many one-loop subdiagrams, but no subdiagrams with two or more loops. These diagrams have the structure of tree diagrams, with one-loop corrections added to the vertices and arbitrarily many oneloop corrections inserted into the propagators. These diagrams can be calculated using the perturbative framework developed in Sec. III. The sum of all such diagrams is independent of the choice of the background field $v(\mathbf{r})$ in Eq. (36). However, the sum of all such diagrams can be greatly simplified by choosing the background field $v$ so that the ground-state expectation values of the quantum fields $\xi$ and $\eta$ vanish. This choice eliminates all one-particle-reducible diagrams that can be disconnected by cutting a single $\xi$ or $\eta$ line. The only diagrams that remain are one-particleirreducible diagrams.

With the Cartesian parametrization (36), the choice of the background field that simplifies self-consistent one-loop calculations is the condensate itself:

$$
v(\mathbf{r})=\langle\psi(\mathbf{r})\rangle .
$$

With this choice, the fields $\xi$ and $\eta$ represent the quantum fluctuations around the ground-state expectation value of $\psi$. Since $v$ is real valued, the expectation value of $\eta$ vanished automatically and the condition (77) can be written

$$
\langle\xi(\mathbf{r})\rangle=0 .
$$

Thus the background field $v$ must be chosen self-consistently so that the quantum fluctuations around that background average to zero. We will refer to the Eq. (78) as the tadpole equation because the one-loop quantum corrections to this equation correspond to Feynman diagrams like those in Figs. 6 and 7 that look like tadpoles. Using the tadpole equation, the density (38) reduces to

$$
\rho(\mathbf{r})=v^{2}(\mathbf{r})+\frac{1}{2}\left\langle\xi^{2}(\mathbf{r})\right\rangle+\frac{1}{2}\left\langle\eta^{2}(\mathbf{r})\right\rangle-\delta \rho .
$$

The ground-state expectation values in Eqs. (78) and (79) are nonlocal functionals of the background $v(\mathbf{r})$. Our strategy is to use the gradient expansion to reduce these functionals to local functions involving $v(\mathbf{r})$ and its derivatives. The tadpole equation (78) then reduces to an algebraic relation between $v(\mathbf{r})$ and its derivatives, while Eq. (79) expresses $\rho$ in terms of $v$ and its derivatives. If we eliminate $v$ from these two equations, we obtain an algebraic relation between $\rho$ and its derivatives. This is the differential equation for $\rho(\mathbf{r})$ that includes self-consistent corrections from one-loop quantum fluctuations.

To calculate the one-loop quantum corrections, we use the decomposition (44) of the action for quantum fluctuations around a general background field $v$. The free part (45) involves only the quantum fields $\xi$ and $\eta$, but introduces an arbitrary scale $\Lambda$. The interaction part (47) involves sources $T, X, Y$, and $Z$ that are given in Eqs. (40)-(43). At one-loop order, the tadpole equation states that the expression (50) for $\langle\xi\rangle$ vanishes, which implies

$$
0=T(\mathbf{r})+\frac{3}{2} Z(\mathbf{r})\left\langle\xi^{2}(\mathbf{r})\right\rangle_{X, Y}+\frac{1}{2} Z(\mathbf{r})\left\langle\eta^{2}(\mathbf{r})\right\rangle_{X, Y} .
$$

Similarly, the expression (79) for the number density reduces at one-loop order to

$$
\rho(\mathbf{r})=v^{2}(\mathbf{r})+\frac{1}{2}\left\langle\xi^{2}(\mathbf{r})\right\rangle_{X, Y}+\frac{1}{2}\left\langle\eta^{2}(\mathbf{r})\right\rangle_{X, Y}-\delta \rho .
$$

Eqs. (80) and (81) are integral equations whose solutions give the condensate and the density in the Hartree-Fock approximation. The quantities $\left\langle\xi^{2}\right\rangle_{X, Y}$ and $\left\langle\eta^{2}\right\rangle_{X, Y}$ in Eqs. (80) and (81) can be expanded in powers of $X$ and $Y$ and their derivatives using Eqs. (51) and (52). Since these expansions include infinitely many terms, the Eqs. (80) and (81) can be reduced to local equations only by consistently truncating the expansions. We will reduce them to local equations at a specific point $\mathbf{r}_{0}$ by (a) using the classical equations for $\rho$ and $v$ to simplify the expressions for the sources, (b) choosing a specific value for the arbitrary parameter $\Lambda$, and (c) truncating the equations at second order in the gradient expansion.

We begin by simplifying the sources $X, Y$, and $Z$ in Eqs. (41), (42), and (43) by using the classical equations $T(\mathbf{r})=0$ and $\rho(\mathbf{r})=v^{2}(\mathbf{r})$. Since $X, Y$, and $Z$ appear only in one-loop diagrams, any quantum corrections to the sources contribute only at second order in the quantum loop expansion. Using $T=0$, we can eliminate the potential $V$ from $X$ and $Y$. Using $v=\sqrt{\rho}$, we can express $X, Y$, and $Z$ in terms of $\rho$ only. We can also simplify $T$ by setting $v=\sqrt{\rho}$ in the terms proportional to the counterterms $\delta \mu$ and $\delta g$. Finally, we can drop the terms in $X$ and $Z$ that involve the counterterm $\delta g$ since it is needed only to cancel ultraviolet divergences that arise at two loops or higher in the quantum loop expansion. Thus the expressions for the sources can be reduced to 


$$
\begin{gathered}
T(\mathbf{r})=\left[\mu-V(\mathbf{r})-\frac{g}{2} v^{2}(\mathbf{r})\right] v(\mathbf{r})+\frac{1}{2 m} \nabla^{2} v(\mathbf{r}) \\
+\left[\delta \mu-\frac{\delta g}{2} \rho(\mathbf{r})\right] \sqrt{\rho}(\mathbf{r}) \\
X(\mathbf{r})=\Lambda^{2}-2 m g \rho(\mathbf{r})-\frac{\nabla^{2} \sqrt{\rho}}{\sqrt{\rho}}(\mathbf{r}), \\
Y(\mathbf{r})=-\frac{\nabla^{2} \sqrt{\rho}}{\sqrt{\rho}}(\mathbf{r}) \\
Z(\mathbf{r})=-\frac{g}{2} \sqrt{\rho}(\mathbf{r}) .
\end{gathered}
$$

Note that the source $Y$ in Eq. (84) is already second order in the gradient expansion. Thus, if we truncate the equations at second order in the gradient expansion, we need only include terms up to first order in $Y$ and we can omit all derivatives of $Y$. We also need only include terms up to first order in $\nabla^{2} X$ and up to second order in $\nabla X$. However, we still must include all possible powers of $X$.

In order to reduce the expansions for Eqs. (80) and (81) to a finite number of terms, we choose $\Lambda$ so that $X\left(\mathbf{r}_{0}\right)$ is second order in the gradient expansion at a specific point $\mathbf{r}_{0}$. A convenient choice for $\Lambda$ is the wave number that appears in the Bogoliubov dispersion relation (32) for a homogeneous Bose gas with number density $\rho\left(\mathbf{r}_{0}\right)$ :

$$
\Lambda^{2}=2 m g \rho\left(\mathbf{r}_{0}\right) .
$$

With this choice for $\Lambda$, the source $X$ and its derivatives at the point $\mathbf{r}_{0}$ reduce to

$$
X\left(\mathbf{r}_{0}\right)=-\frac{\nabla^{2} \sqrt{\rho}}{\sqrt{\rho}}\left(\mathbf{r}_{0}\right),
$$

$$
\begin{gathered}
\boldsymbol{\nabla} X\left(\mathbf{r}_{0}\right)=-4 m g \sqrt{\rho} \boldsymbol{\nabla} \sqrt{\rho}\left(\mathbf{r}_{0}\right), \\
\nabla^{2} X\left(\mathbf{r}_{0}\right)=-4 m g\left[\sqrt{\rho} \nabla^{2} \sqrt{\rho}+(\nabla \sqrt{\rho})^{2}\right]\left(\mathbf{r}_{0}\right) .
\end{gathered}
$$

We proceed to determine the differential equation satisfied by $\rho$ in the self-consistent one-loop approximation. This equation can be obtained by solving Eq. (81) for the condensate $v$ in terms of the density $\rho$ and its derivatives and then eliminating $v$ from the tadpole equation (80). If the tadpole equation is evaluated at the point $\mathbf{r}_{0}$ and then truncated at second order in the gradient expansion, it reduces to

$$
\begin{aligned}
0= & T\left(\mathbf{r}_{0}\right)+Z\left(\mathbf{r}_{0}\right)\left[\frac{3 a_{0}+b_{0}}{2}+\frac{3 a_{1}+b_{1}}{2} X\left(\mathbf{r}_{0}\right)\right. \\
& +\frac{3 a_{2}+b_{2}}{2} Y\left(\mathbf{r}_{0}\right)+\frac{3 a_{3}+b_{3}}{2} \nabla^{2} X\left(\mathbf{r}_{0}\right) \\
& \left.+\frac{3 a_{5}+b_{5}}{2}(\nabla X)^{2}\left(\mathbf{r}_{0}\right)\right] .
\end{aligned}
$$

The expression (82) for the source $T$ involves $v$ and $\nabla^{2} v$. Solving Eq. (81) for $v(\mathbf{r})$ to first order in the quantum corrections, we obtain

$$
\begin{aligned}
v(\mathbf{r})= & \sqrt{\rho}(\mathbf{r})-\frac{1}{2 \sqrt{\rho}(\mathbf{r})}\left[\frac{a_{0}+b_{0}-2 \delta \rho}{2}+\frac{a_{1}+b_{1}}{2} X(\mathbf{r})\right. \\
& +\frac{a_{2}+b_{2}}{2} Y(\mathbf{r})+\frac{a_{3}+b_{3}}{2} \nabla^{2} X(\mathbf{r})+\frac{a_{4}+b_{4}}{2} X^{2}(\mathbf{r}) \\
& \left.+\frac{a_{5}+b_{5}}{2}(\nabla X)^{2}(\mathbf{r})+\cdots\right] .
\end{aligned}
$$

We then substitute this expression for $v$ into the source $T(\mathbf{r})$ in Eq. (82) and expand to first order in quantum fluctuations. After calculating the derivative $\nabla^{2} v$ appearing in $T$, we can set $\mathbf{r}=\mathbf{r}_{0}$ and then truncate at second order in the gradient expansion. Using the expressions (84) and (87)-(89) for the sources and their derivatives, the expression for $T\left(\mathbf{r}_{0}\right)$ reduces to

$$
\begin{aligned}
T= & \left(\mu-V-\frac{g}{2} \rho\right) \sqrt{\rho}+\frac{1}{2 m} \nabla^{2} \sqrt{\rho}+\left(\frac{a_{0}+b_{0}-2 \delta \rho}{4}+\frac{\delta \mu}{g}\right) g \sqrt{\rho}-\frac{\delta g}{2} \rho \sqrt{\rho} \\
& +\left(\frac{a_{0}+b_{0}-2 \delta \rho}{4}+\frac{a_{1}+b_{1}-a_{2}-b_{2}}{8} \Lambda^{2}-\frac{a_{3}+b_{3}}{4} \Lambda^{4}\right) \frac{\nabla^{2} \sqrt{\rho}}{m \rho} \\
& -\left(\frac{a_{0}+b_{0}-2 \delta \rho}{4}+\frac{a_{1}+b_{1}}{4} \Lambda^{2}+\frac{a_{3}+b_{3}+4 a_{4}+4 b_{4}}{4} \Lambda^{4}-\frac{a_{5}+b_{5}}{2} \Lambda^{6}\right) \frac{(\nabla \sqrt{\rho})^{2}}{m \rho \sqrt{\rho}} \\
& +(v-\sqrt{\rho})\left[\mu-V-\frac{g}{2} \rho+\frac{1}{2 m} \frac{\nabla^{2} \sqrt{\rho}}{\sqrt{\rho}}\right]
\end{aligned}
$$

where we have used the expression (86) for $\Lambda$. The last term in Eq. (92) can be dropped because it is proportional to the classical equation (1). Its effects are therefore of second order in the quantum loop expansion. This eliminates all occurrences of the potential $V$ in the quantum corrections. Inserting the resulting expression for $T\left(\mathbf{r}_{0}\right)$ into Eq. (90), the tadpole equation reduces to 


$$
\begin{aligned}
0= & \left(\mu-V-\frac{g}{2} \rho\right) \sqrt{\rho}+\frac{1}{2 m} \nabla^{2} \sqrt{\rho}-\left(\frac{a_{0}+\delta \rho}{2}-\frac{\delta \mu}{g}\right) g \sqrt{\rho}-\frac{\delta g}{2} \rho \sqrt{\rho} \\
& +\left(\frac{a_{0}+b_{0}-2 \delta \rho}{4}+\frac{2 a_{1}+b_{1}+a_{2}}{4} \Lambda^{2}+\frac{a_{3}}{2} \Lambda^{4}\right) \frac{\nabla^{2} \sqrt{\rho}}{m \rho} \\
& -\left(\frac{a_{0}+b_{0}-2 \delta \rho}{4}+\frac{a_{1}+b_{1}}{4} \Lambda^{2}-\frac{a_{3}-2 a_{4}-2 b_{4}}{2} \Lambda^{4}+a_{5} \Lambda^{6}\right) \frac{(\nabla \sqrt{\rho})^{2}}{m \rho \sqrt{\rho}} .
\end{aligned}
$$

Using the results for the coefficients $a_{i}$ and $b_{i}$ given in Appendix $\mathrm{C}$ and using Eq. (86) to set $\Lambda^{2}=2 m g \rho\left(\mathbf{r}_{0}\right)$, the equation for $\rho$ reduces to

$$
\begin{aligned}
0= & {[\mu-V(\mathbf{r})] \sqrt{\rho}(\mathbf{r})-\frac{g}{2} \rho \sqrt{\rho}(\mathbf{r})+\frac{1}{2 m} \nabla^{2} \sqrt{\rho}(\mathbf{r}) } \\
& -\frac{1}{48 \pi^{2}}(2 m g)^{3 / 2}\left[4 g \rho^{2}(\mathbf{r})+\frac{17}{24 m}\left[2 \sqrt{\rho} \nabla^{2} \sqrt{\rho}(\mathbf{r})\right.\right. \\
& \left.\left.+(\boldsymbol{\nabla} \sqrt{\rho})^{2}(\mathbf{r})\right]\right] .
\end{aligned}
$$

We derived this equation at the point $\mathbf{r}_{0}$ defined by our choice (86) for the arbitrary parameter $\Lambda$. However, our final result is an algebraic equation relating $\sqrt{\rho}$ and its derivatives at the point $\mathbf{r}_{0}$. Since we could have chosen any specific point for $\mathbf{r}_{0}$, this algebraic relation must hold at any point $\mathbf{r}$. Using Eq. (6) to eliminate $g$ in favor of $a$ and using dimensional analysis to insert the appropriate factors of $\hbar$ into our Eq. (94), we obtain the differential equation (2) for the density profile.

The $\rho^{2}$ term in Eq. (94) can be obtained from previous work on the homogeneous Bose gas. Differentiating the result (31) for the energy density with respect to $\sqrt{\rho}$, we obtain

$$
\frac{\partial \mathcal{E}}{\partial \sqrt{\rho}}=g \rho \sqrt{\rho}\left[1+\frac{1}{6 \pi^{2}}(2 m g)^{3 / 2} \sqrt{\rho}\right] .
$$

Multiplying by $-1 / 2$, we reproduce the $\rho \sqrt{\rho}$ and $\rho^{2}$ terms in Eq. (94). The $\sqrt{\rho} \nabla^{2} \sqrt{\rho}$ and $(\nabla \sqrt{\rho})^{2}$ terms in Eq. (94) are new results.

As a check of the Eq. (94), we can verify that our oneloop expression for $\rho$ given in Eq. (74) satisfies Eq. (94) after expanding to first order in the quantum fluctuations. There is an important qualitative difference between the approximate solution (74) and the solution to the self-consistent equation (94). The solution to Eq. (94) has the correct qualitative behavior even outside the condensate. In this region, the density is very small and only the terms in Eq. (94) that are linear in $\sqrt{\rho}$ are important. The equation therefore reduces to

$$
0 \approx[\mu-V(\mathbf{r})] \sqrt{\rho}(\mathbf{r})+\frac{1}{2 m} \nabla^{2} \sqrt{\rho}(\mathbf{r}) .
$$

The quantum correction terms in Eq. (94) were calculated using a gradient expansion that is valid only inside the condensate. However, since these terms are all higher order in $\sqrt{\rho}$, their effects are negligible outside the condensate and it does no harm to include them. In contrast, the approximate solution (74) has the wrong qualitative behavior when $\phi_{0}$ is small because it is dominated by the $\nabla^{2} \phi_{0}$ and $\left(\nabla \phi_{0}\right)^{2} / \phi_{0}$ terms. Thus that solution can only be used inside the condensate.

\section{POLAR FIELD PARAMETRIZATION}

The one-loop calculations in Secs. IV and V were carried out using the Cartesian parametrization of the quantum field given in Eq. (36). There is nothing special about this parametrization aside from its simplicity. Other field parametrizations should give the same final result for physical quantities. An example of an alternative field parametrization is the polar parametrization

$$
\psi(\mathbf{r}, t)=\sqrt{v^{2}(\mathbf{r})+\sigma(\mathbf{r}, t)} \exp [i \alpha(\mathbf{r}, t)] .
$$

The advantage of this parametrization is that it eliminates infrared divergences from individual Feynman diagrams that contribute to the number density. In this section we verify that the polar parametrization gives the same equation for the density profile. We also use this parametrization to show that the gradient expansion of the density breaks down at fourth order.

With the polar parametrization (97), the choice for the background field $v$ that simplifies self-consistent one-loop quantum corrections is the one specified by the tadpole equation

$$
\langle\sigma(\mathbf{r})\rangle=0 .
$$

The expression (22) for the number density reduces to

$$
\rho(\mathbf{r})=v^{2}(\mathbf{r})-\delta \rho .
$$

Thus the choice of the background $v(\mathbf{r})$ implied by the tadpole condition (98) is

$$
v(\mathbf{r})=\sqrt{\rho(\mathbf{r})}+\frac{\delta \rho}{2} \frac{1}{\sqrt{\rho(\mathbf{r})}}+\cdots .
$$

Since this expression involves the ultraviolet divergent constant $\delta \rho, v$ has no simple physical interpretation. It is best regarded as a theoretical construct that should appear only in intermediate stages of a calculation. The simplicity of the expression (99) for $\rho$ comes at the expense of the expression for the condensate. Expanding Eq. (97) as a power series in $\sigma$ and $\alpha$ and taking the ground-state expectation value, we obtain 


$$
\langle\psi(\mathbf{r})\rangle=v(\mathbf{r})-\frac{1}{8 v^{3}(\mathbf{r})}\left\langle\sigma^{2}(\mathbf{r})\right\rangle-\frac{v(\mathbf{r})}{2}\left\langle\alpha^{2}(\mathbf{r})\right\rangle+\cdots .
$$

The expansion (101) includes infinitely many terms and we have written explicitly only those terms that contribute at one-loop order. The expectation values of operators involving four or more powers of $\sigma$ or $\alpha$ contribute at two-loop order or higher.

We begin our calculation by inserting the parametrization (97) into the action (21) and expanding in powers of the quantum fields $\sigma$ and $\alpha$ :

$$
\begin{aligned}
S[\psi]= & S[v]+\int d t \int d^{3} r\left\{\frac{1}{v} T \sigma+\frac{1}{2}(\alpha \dot{\sigma}-\sigma \dot{\alpha})\right. \\
& -\frac{v^{2}}{2 m}(\boldsymbol{\nabla} \alpha)^{2}-\frac{1}{8 m v^{2}}(\nabla \sigma)^{2}-\left(\frac{g+\delta g}{4}+\frac{\nabla^{2} v}{4 m v^{3}}\right. \\
& \left.-\frac{(\nabla v)^{2}}{4 m v^{4}}\right) \sigma^{2}-\frac{1}{2 m} \sigma(\nabla \alpha)^{2}+\frac{1}{8 m v^{4}} \sigma(\nabla \sigma)^{2} \\
& \left.+\left(\frac{\nabla^{2} v}{6 m v^{5}}-\frac{(\nabla v)^{2}}{3 m v^{6}}\right) \sigma^{3}+\cdots\right\},
\end{aligned}
$$

where $T$ is the external source given in Eq. (40). The parametrization (97) leads to an infinite series of momentumdependent interactions. We have dropped terms that are fourth and higher order in the quantum fields since they do not contribute to the one-loop quantum corrections to the density profile. It is convenient to introduce an arbitrary parameter $\Lambda$ into the action by rescaling the quantum fields as follows:

$$
\begin{aligned}
& \sigma(\mathbf{r}, t)=\frac{\Lambda}{\sqrt{m g}} \xi(\mathbf{r}, t), \\
& \alpha(\mathbf{r}, t)=\frac{\sqrt{m g}}{\Lambda} \eta(\mathbf{r}, t) .
\end{aligned}
$$

After these rescalings, we separate the action into a free part and an interaction part as in Eq. (44). The free part is identical to Eq. (45) and the interaction part is

$$
\begin{aligned}
S_{\text {int }}[v, \xi, \eta]= & \int d t \int d^{3} r\left\{\sqrt{2} \frac{\Lambda}{\sqrt{2 m g v^{2}}} T \xi+\frac{1}{4 m} X \xi^{2}\right. \\
& +\frac{1}{4 m} U(\boldsymbol{\nabla} \xi)^{2}+\frac{1}{4 m} S(\boldsymbol{\nabla} \eta)^{2} \\
& +\frac{1}{\sqrt{2}} Z \xi^{3}+\frac{1}{\sqrt{2} W \xi(\nabla \xi)^{2}} \\
& \left.-\frac{1}{\sqrt{8} m v} \frac{\sqrt{2 m g v^{2}}}{\Lambda} \xi(\nabla \eta)^{2}+\cdots\right\}, \quad
\end{aligned}
$$

where $X, Z, U, S$, and $W$ are external sources that depend on the background $v$ :

$$
X(\mathbf{r})=-2 \frac{\Lambda^{2}}{2 m g v^{2}(\mathbf{r})}\left[\frac{\nabla^{2} v}{v}-\frac{(\nabla v)^{2}}{v^{2}}\right](\mathbf{r})-\frac{\delta g}{g} \Lambda^{2},
$$

$$
\begin{gathered}
Z(\mathbf{r})=\frac{2}{3 m v(\mathbf{r})}\left(\frac{\Lambda^{2}}{2 m g v^{2}(\mathbf{r})}\right)^{3 / 2}\left[\frac{\nabla^{2} v}{v}-2 \frac{(\boldsymbol{\nabla} v)^{2}}{v^{2}}\right](\mathbf{r}), \\
U(\mathbf{r})=1-\frac{\Lambda^{2}}{2 m g v^{2}(\mathbf{r})} \\
S(\mathbf{r})=1-\frac{2 m g v^{2}(\mathbf{r})}{\Lambda^{2}} \\
W(\mathbf{r})=\frac{1}{2 m v(\mathbf{r})}\left(\frac{\Lambda^{2}}{2 m g v^{2}(\mathbf{r})}\right)^{3 / 2}
\end{gathered}
$$

The arbitrary parameter $\Lambda$, which was introduced through the rescaling of the fields, appears in both the free part of the action and the interactions. We will exploit the arbitrariness of this parameter to simplify the calculation of quantum corrections.

The tadpole equation (98) can be written $\langle\xi(\mathbf{r})\rangle=0$. To first order in the quantum corrections, this equation implies that $\left(\Lambda / \sqrt{2 m g v^{2}}\right) T$ plus the sum of all one-loop tadpole diagrams vanishes. The one-loop diagrams include all possible insertions of the sources $X, U$, and $S$. The one-loop tadpole equation can be written succinctly in the form

$$
\begin{aligned}
0= & \frac{\Lambda}{\sqrt{2 m g v^{2}}} T+\frac{3}{2} Z\left\langle\xi^{2}\right\rangle_{X, U, S}-\frac{1}{2} \nabla \cdot\left[W \nabla\left\langle\xi^{2}\right\rangle_{X, U, S}\right] \\
& +\frac{1}{2} W\left\langle(\nabla \xi)^{2}\right\rangle_{X, U, S}-\frac{1}{4 m v} \frac{\sqrt{2 m g v^{2}}}{\Lambda}\left\langle(\nabla \eta)^{2}\right\rangle_{X, U, S},
\end{aligned}
$$

where \langle\rangle$_{X, U, S}$ denotes the ground-state expectation value in the presence of the sources $X, U$, and $S$, but with no other interactions. The expectation values in Eq. (111) are nonlocal functionals of these sources. After Taylor expanding the sources around the point $\mathbf{r}$, these functionals can be expanded in powers of $X, U$, and $S$ and their derivatives at the point $\mathbf{r}$ :

$$
\begin{aligned}
\left\langle\xi^{2}\right\rangle_{X, U, S}=c_{0}+ & c_{1} U+c_{2} S+c_{3} U^{2}+c_{4} U S+c_{5} S^{2}+\cdots \\
\left\langle(\nabla \xi)^{2}\right\rangle_{X, U, S}= & d_{0}+d_{1} X+d_{2} \nabla^{2} U+d_{3} \nabla^{2} S+d_{4}(\nabla U)^{2} \\
& +d_{5} \nabla U \cdot \nabla S+d_{6}(\nabla S)^{2}+\cdots \\
\left\langle(\nabla \eta)^{2}\right\rangle_{X, U, S}= & e_{0}+e_{1} X+e_{2} \nabla^{2} U+e_{3} \nabla^{2} S+e_{4}(\nabla U)^{2} \\
& +e_{5} \boldsymbol{\nabla} U \cdot \nabla S+e_{6}(\nabla S)^{2}+\cdots
\end{aligned}
$$

The terms on the right-hand sides of Eqs. (112)-(114) include all combinations of $X, U, S$, and their derivatives that are allowed by rotational symmetry. We have written explicitly only those terms that are required to calculate the equation for the density through second order in the gradient expansion.

The right-hand side of Eq. (111) is a nonlocal functional of $v$. When it is expanded in powers of $X, U, S$, and their derivatives, there are infinitely many terms. The equation can be reduced to a local one only by consistently truncating the expansions. We will reduce Eq. (111) to a local equation at a specific point $\mathbf{r}_{0}$ by (a) choosing a specific value for the 
arbitrary parameter $\Lambda$ and (b) truncating the equations at second order in the gradient expansion. Since the sources $X$ and $Z$ in Eqs. (106) and (107) are already second order in the gradient expansion, we need only keep terms of first order in $X$ and $Z$ and we can omit any derivatives of $X$. Moreover, we need only include terms that are first order in $\nabla^{2} U$ and $\nabla^{2} S$ and terms up to second order in $\boldsymbol{\nabla} U$ and $\boldsymbol{\nabla} S$. However, Eq. (111) still includes all possible powers of $U$ and $S$. We can reduce this equation to a finite number of terms at a specific point $\mathbf{r}_{0}$ by choosing $\Lambda$ so that the sources $U$ and $S$ vanish at the point $\mathbf{r}_{0}$. The required value is

$$
\Lambda^{2}=2 m g v^{2}\left(\mathbf{r}_{0}\right) .
$$

With this choice of $\Lambda$, the sources on the right-hand sides of Eqs. (112)-(114) reduce to

$$
\begin{gathered}
X\left(\mathbf{r}_{0}\right)=-2\left[\frac{\nabla^{2} v}{v}-\frac{(\boldsymbol{\nabla} v)^{2}}{v^{2}}\right]\left(\mathbf{r}_{0}\right), \\
U\left(\mathbf{r}_{0}\right)=0, \\
\boldsymbol{\nabla} U\left(\mathbf{r}_{0}\right)=2 \frac{\boldsymbol{\nabla} v}{v}\left(\mathbf{r}_{0}\right), \\
\nabla^{2} U\left(\mathbf{r}_{0}\right)=2\left[\frac{\nabla^{2} v}{v}-3 \frac{(\boldsymbol{\nabla} v)^{2}}{v^{2}}\right]\left(\mathbf{r}_{0}\right), \\
\nabla\left(\mathbf{r}_{0}\right)=0, \\
\nabla^{2} S\left(\mathbf{r}_{0}\right)=-2\left[\frac{\left.\left.\mathbf{r}_{0}\right)=-2 \frac{\nabla^{2} v}{v}+\frac{\left(\mathbf{r}_{0}\right)}{v^{2}}\right]}{2}\right]\left(\mathbf{r}_{0}\right),
\end{gathered}
$$

In the expression (116) for $X\left(\mathbf{r}_{0}\right)$, we have dropped the term involving the counterterm $\delta g$ since it is needed only to can- cel ultraviolet divergences that arise at two loops or higher. With the choice (115) for $\Lambda$, the tadpole equation (111) simplifies at the point $\mathbf{r}_{0}$ to

$$
\begin{aligned}
0= & T+\frac{1}{m}\left[\frac{\nabla^{2} v}{v^{2}}-2 \frac{(\boldsymbol{\nabla} v)^{2}}{v^{3}}\right]\left\langle\xi^{2}\right\rangle_{X, U, S}-\frac{1}{4 m v} \nabla^{2}\left\langle\xi^{2}\right\rangle_{X, U, S} \\
& +\frac{\boldsymbol{\nabla} v}{m v^{2}} \cdot \nabla\left\langle\xi^{2}\right\rangle_{X, U, S}+\frac{1}{4 m v}\left[\left\langle(\nabla \xi)^{2}\right\rangle_{X, U, S}\right. \\
& \left.-\left\langle(\boldsymbol{\nabla} \eta)^{2}\right\rangle_{X, U, S}\right] .
\end{aligned}
$$

After inserting the expansions (112)-(114) into the tadpole equation (123) and evaluating it at $\mathbf{r}_{0}$, we can truncate it at second order in the gradient expansion. Using the expressions (116)-(122) for the sources, we obtain an algebraic equation relating $v, \nabla v$, and $\nabla^{2} v$ at the point $\mathbf{r}_{0}$. To express this equation in terms of $\rho$ and its derivatives, we eliminate $v$ using Eq. (100). Since the term $\delta \rho / 2 \sqrt{\rho}$ in Eq. (100) is first order in quantum fluctuations, it is needed only in the term $T$ in Eq. (123). The source $T$ then becomes

$$
\begin{aligned}
T= & \left(\mu-V-\frac{g}{2} \rho\right) \sqrt{\rho}+\frac{1}{2 m} \nabla^{2} \sqrt{\rho}-\left(\frac{\delta \rho}{2}-\frac{\delta \mu}{g}\right) g \sqrt{\rho} \\
& -\frac{\delta g}{2} \rho \sqrt{\rho}-\frac{\delta \rho}{2}\left(\frac{\nabla^{2} \sqrt{\rho}}{m \rho}-\frac{(\nabla \sqrt{\rho})^{2}}{m \rho \sqrt{\rho}}\right) \\
& +\frac{\delta \rho}{2}\left[\left(\mu-V-\frac{g}{2} \rho\right) \frac{1}{\sqrt{\rho}}+\frac{1}{2 m} \frac{\nabla^{2} \sqrt{\rho}}{\rho}\right] .
\end{aligned}
$$

The last term in Eq. (124) can be dropped because it is proportional to the classical equation (1). Its effects are therefore second order in the quantum loop expansion. After inserting Eq. (124) into Eq. (123), the tadpole equation reduces to

$$
\begin{aligned}
0= & \left(\mu-V-\frac{g}{2} \rho\right) \sqrt{\rho}+\frac{1}{2 m} \nabla^{2} \sqrt{\rho}+\frac{d_{0}-e_{0}}{4} \frac{1}{m \sqrt{\rho}}+\left(\frac{\delta \mu}{g}-\frac{\delta \rho}{2}\right) g \sqrt{\rho}-\frac{\delta g}{2} \rho \sqrt{\rho} \\
& +\left(c_{0}-\frac{c_{1}-c_{2}}{2}-\frac{d_{1}-d_{2}+d_{3}}{2}+\frac{e_{1}-e_{2}+e_{3}}{2}-\frac{\delta \rho}{2}\right) \frac{\nabla^{2} \sqrt{\rho}}{m \rho} \\
& -\left(2 c_{0}-\frac{7 c_{1}-3 c_{2}}{2}+2\left(c_{3}-c_{4}+c_{5}\right)-\frac{d_{1}-3 d_{2}-d_{3}}{2}-\left(d_{4}-d_{5}+d_{6}\right)+\frac{e_{1}-3 e_{2}-e_{3}}{2}+\left(e_{4}-e_{5}+e_{6}\right)-\frac{\delta \rho}{2}\right) \frac{(\nabla \sqrt{\rho})^{2}}{m \rho \sqrt{\rho}} .
\end{aligned}
$$

The coefficients $c_{i}, d_{i}$, and $e_{i}$ are given in Appendix C. The coefficients are all infrared finite but ultraviolet divergent. The ultraviolet divergences from individual diagrams are more severe than those encountered with the Cartesian field parametrization used in Secs. IV and V. The integrals $d_{0}$ and $e_{0}$ diverge as the fifth power of the ultraviolet cutoff, but they cancel in the combination $d_{0}-e_{0}$. The remaining ultraviolet divergences are canceled by the counterterms $\delta \mu, \delta g$, and $\delta \rho$, whose values are given in Eqs. (68), (69), and (73).
Using the expression for the coefficients given in Appendix C, Eq. (125) reduces to

$$
\begin{aligned}
0= & (\mu-V) \sqrt{\rho}-\frac{g}{2} \rho \sqrt{\rho}+\frac{1}{2 m} \nabla^{2} \sqrt{\rho} \\
& -\frac{1}{48 \pi^{2}}\left[2 \frac{\Lambda^{5}}{m \sqrt{\rho}}+\frac{17}{12} \frac{\Lambda^{3}}{m \rho} \nabla^{2} \sqrt{\rho}+\frac{17}{24} \frac{\Lambda^{3}}{m \rho \sqrt{\rho}}(\nabla \sqrt{\rho})^{2}\right] .
\end{aligned}
$$


After using Eq. (115) to set $\Lambda^{2}=2 m g \rho\left(\mathbf{r}_{0}\right)$, we reproduce the self-consistent one-loop equation (94) for the density profile.

There has been a previous attempt to calculate the quantum corrections to the Gross-Pitaevskii equation. ${ }^{21}$ The authors used the polar field parametrization (97), with the background field $v(\mathbf{r})$ equal to the mean field $\phi_{0}(\mathbf{r})$. They dropped all terms in the action that were third order and higher in $\alpha$ and $\sigma$ and they also dropped second-order terms that involved gradients of $\phi_{0}$ or $\sigma$. The only terms remaining in the action that contribute to the density profile are

$$
\begin{aligned}
S[\psi]= & S\left[\phi_{0}\right]+\int d t \int d^{3} r\left\{\frac{1}{2}(\alpha \dot{\sigma}-\sigma \dot{\alpha})\right. \\
& \left.-\frac{\phi_{0}^{2}(\mathbf{r})}{2 m}(\nabla \alpha)^{2}-\frac{g}{4} \sigma^{2}\right\} .
\end{aligned}
$$

With such a drastic truncation of the action, the quantum corrections that they ultimately calculate are of no relevance to the problem of atoms in a trapping potential. This is evident from the fact that the Bogoliubov dispersion relation (32) never enters into the quantum corrections that they calculate. Thus their approach is incapable of reproducing the known results for a homogeneous Bose gas.

A comparison of the calculation above with that presented in Sec. V demonstrates that the Cartesian field parametrization is more efficient than the polar field parametrization for explicit calculations. With the polar field parametrization, one avoids infrared divergent integrals at intermediate stages of the calculation, but this advantage is compensated by the fact that the integrals are more severely ultraviolet divergent. The simplicity of the relation (99) between $\rho$ and $v$ is compensated by a tadpole equation (111) that is more complicated than the corresponding equation (80) in the Cartesian field parametrization.

The advantage of the polar field parametrization is that it avoids cancellations of infrared divergences between different diagrams. This makes it easier to identify the sources of infrared divergences that are responsible for the breakdown of the gradient expansion. We will use this parametrization to show that the gradient expansion of the density breaks down at fourth order. The component of the propagator matrix (46) that is most infrared sensitive is $D^{\eta \eta}$. For small loop momentum $k$, the frequency $\omega$ in the loop scales like $\Lambda k / 2 m$ and $D^{\eta \eta}$ scales like $2 m / k^{2}$. The most infrared singular diagrams are those for which all the lines are $\eta$ lines. The term in the tadpole equation (111) that is most infrared sensitive is $\left\langle(\boldsymbol{\nabla} \eta)^{2}\right\rangle_{X, U, S}$, because the operator $(\boldsymbol{\nabla} \eta)^{2}$ creates two $\eta$ lines. In the expansion (114) for that matrix element, the most infrared singular terms are those that involve only the source $S$, which couples to a pair of $\eta$ lines. The infrared behavior of the coefficient of a term in Eq. (114) that involves $m$ factors of $\boldsymbol{\nabla}$ and $n$ factors of $S$ can be determined by simple power counting. The integrand has a factor of $1 / k^{2}$ for each of the $n+1$ propagators. There is a factor of $k^{2}$ for the operator $(\nabla \eta)^{2}$ and a factor of $k^{2}$ for each insertion of $S$. Finally, dimensional analysis requires that each factor of $\boldsymbol{\nabla}$ be compensated by a factor of $1 / k$ in the integrand. Thus the integral must scale like

$$
\int d \omega \int d^{3} k k^{2}\left(\frac{1}{k^{2}}\right)^{n+1}\left(k^{2}\right)^{n}\left(\frac{1}{k}\right)^{m} \sim \int d k k^{3-m} .
$$

An infrared divergence can appear only if $m \geqslant 4$. Thus infrared divergences first appear in the tadpole equation when it is expanded to fourth order in the gradient expansion.

Our explicit one-loop calculation of $\langle\psi\rangle$ in Eq. (70) showed that the gradient expansion for the condensate breaks down at second order. The analysis presented above shows that the gradient expansion for the density $\rho$ does not break down until fourth order. The breakdown of the gradient expansion implies that the quantum corrections depend on nonlocal effects involving the length scale $L$ for significant variations in $\rho$. While we have identified the orders at which the gradient expansions break down, we have not identified any deep reason for the gradient expansion of the density to be better behaved than that of the condensate.

\section{IMPLICATIONS FOR PRESENT TRAPS}

In this section we estimate the magnitude of the effects of quantum fluctuations for Bose-Einstein condensates in existing magnetic traps. For numerical estimates, we will use parameters characteristic of the sodium experiment, ${ }^{3}$ which has achieved the highest density condensates to date. In this experiment, $N \approx 5 \times 10^{6}$ sodium atoms were condensed in a trapping potential with a length scale $\ell \approx 2 \mu \mathrm{m}$. The $S$-wave scattering length for sodium atoms is $a \approx 0.005 \mu \mathrm{m}$. The number density that was attained at the center of the condensate was $\rho \approx 400 / \mu \mathrm{m}^{3}$.

Baym and Pethick have presented a simple qualitative analysis of the solution to the Gross-Pitaevskii equation that allows one to determine how various quantities scale with the number $N$ of atoms. ${ }^{6}$ The qualitative behavior of the solution to Eq. (1) depends crucially on a dimensionless parameter $\zeta$ given by

$$
\zeta=\left(\frac{8 \pi N a}{\ell}\right)^{1 / 5}
$$

where $\ell$ is the length scale associated with significant variations in the potential $V(\mathbf{r})$. For a harmonic oscillator potential, $\ell=\hbar / \sqrt{m \omega}$, where $\omega$ is the angular frequency of the harmonic oscillator. If $\zeta$ is less than or of order 1 , the size of the condensate is comparable to $\ell$ and the number density inside the trap scales like $N / \ell^{3}$. If $\zeta$ is much greater than 1 , the size $L$ of the condensate scales like $L=\zeta \ell$ and the density inside the trap scales like $N /(\zeta \ell)^{3}$. The condensates in existing magnetic traps are characterized by values of $\zeta$ that are significantly greater than 1 . (For the sodium experiment, $\zeta \approx 13$.) We will determine how the correction terms in our equation for the density profile scale with $N$ in the case $\zeta \gg 1$. The expansion parameter $\sqrt{\rho a^{3}}$ for the low-density expansion scales like $\zeta a / \ell$. Although $\zeta$ is large for existing traps, $a / \ell$ is tiny and the product $\zeta a / \ell$ is small. (For the sodium experiment, $\zeta a / \ell \approx 0.03$.) The modes that dominate the quantum corrections have wavelengths on the order of $1 / \sqrt{\rho a}$, which scales like $\ell / \zeta$. Since this is small compared to the length scale $\zeta \ell$ for significant variations in $\rho(\mathbf{r})$, it is reasonable to expand the quantum corrections using the gra- 
dient expansion. The gradient expansion corresponds to an expansion in powers of the dimensionless quantity $1 / \sqrt{\rho a} \zeta \ell$, which scales like $1 / \zeta^{2}$. Thus, inside the condensate, quantum corrections are suppressed by $\zeta a / \ell$ and corrections from second order in the gradient expansion are suppressed by $1 / \zeta^{4}$.

Outside the condensate, the density $\rho$ rapidly approaches 0 . The only terms in Eq. (2) that are important in this region are the terms that are linear in $\sqrt{\rho}$. The scale of the gradient is now set by the length scale $\ell$ for significant variations in $V(\mathbf{r})$. In this region, the basic assumption underlying our calculation, that the quantum corrections are dominated by wavelengths of order $1 / \sqrt{\rho a}$, breaks down completely. However, all the quantum corrections terms are higher order in $\sqrt{\rho}$ and therefore have a negligible effect on the solution outside the condensate. Thus it does no harm to include the quantum correction terms in Eq. (2) in the exterior region.

The crossover region between the interior and exterior of the condensate can be characterized by the fact that the $\nabla^{2} \sqrt{\rho}$ term and the $\rho \sqrt{\rho}$ terms become comparable in importance. The gradient expansion breaks down in this region. At the beginning of the crossover region, $\boldsymbol{\nabla}$ still scales like $1 / \zeta \ell$, but the density has decreased to the point that $\rho$ scales like $a /(\zeta \ell)^{2}$. Therefore, the quantum loop expansion parameter $\sqrt{\rho a^{3}}$ scales like $a / \zeta \ell$. As long as this quantity is sufficiently small, the quantum correction terms in Eq. (2) are negligible. (In the sodium experiment, we have $a / \zeta \ell \approx 0.0002$.) Thus it does no harm to include the quantum correction terms in Eq. (2) in the crossover region. We conclude that the differential equation (2) can be used to calculate the density profile everywhere.

We now give a quantitative estimate of the error from truncating the quantum loop expansion at one-loop order. A simple estimate of the relative magnitude of the quantum corrections is the ratio of the $\rho^{2}$ correction term in Eq. (2) to the $\rho \sqrt{\rho}$ term, which is $(32 / 3) \sqrt{\rho a^{3} / \pi}$. For the sodium experiment, this ratio is approximately 0.04 at the center of the condensate. This is small enough that quantum corrections can be treated as small perturbation to the mean-field approximation. Since $\sqrt{\rho a^{3}}$ scales like $N^{1 / 5}$, the number of atoms in the trap could be increased by many orders of magnitude and the condensate would still be within the perturbative region.

A naive estimate of the relative magnitude of two-loop quantum corrections is the square of the magnitude of the one-loop quantum corrections. Their effects should therefore be negligible. One complication is that the two-loop correction depends not only on the $S$-wave scattering length $a$, but also on a second parameter that represents a pointlike contribution to the $3 \rightarrow 3$ scattering amplitude. ${ }^{16}$ If this parameter is anomalously large, the two-loop quantum corrections could be significantly larger than the naive estimate.

We next quantify the errors from truncating the equation for the density at second order in the gradient expansion. A simple estimate of the relative magnitude of contributions from second order in the gradient expansion is the ratio of the $\nabla^{2} \sqrt{\rho}$ term to the $\rho \sqrt{\rho}$ term in the Gross-Pitaevskii equation (1). Assuming that $\nabla^{2} \sqrt{\rho}$ scales like $\sqrt{\rho} /(\zeta \ell)^{2}$, the ratio is $1 / 8 \rho a\left(\zeta \ell^{2}\right)$. In the sodium experiment, this ratio is roughly $10^{-5}$ at the center of the condensate. These correc- tions are therefore negligible. The relative importance of quantum corrections that are second order in the gradient expansion increases as one approaches the edge of the condensate, where the gradient expansion breaks down. However they are still suppressed by a quantum loop factor of order $a / \zeta \ell$.

The gradient expansion for the density breaks down at fourth order, but the breakdown in only logarithmic in $8 \rho a(\zeta \ell)^{2}$. We can estimate the magnitude of these corrections by taking the logarithms to be of order 1 . These corrections are therefore suppressed by two powers of $1 / 8 \rho a(\zeta \ell)^{2}$. There is also an additional suppression factor of $\sqrt{\rho a^{3}}$, since terms of fourth order in the gradient expansion enter only through quantum corrections. Thus these corrections should be completely negligible.

\section{CONCLUSION}

In this paper we have developed a framework for calculating the dominant effects of quantum fluctuations in a Bose-Einstein condensate trapped by an external potential. Our method is based on a combination of the Hartree-Fock approximation and an expansion around the Thomas-Fermi limit. We have illustrated the method by calculating the selfconsistent one-loop equation for the density profile to second order in the gradient expansion and the relation between the condensate and the density to zeroth order in the gradient expansion. It should be straightforward to use this method to calculate the effects of quantum fluctuations on other properties of the condensate at zero temperature, such as the spectrum of its collective excitations. It would also be interesting to extend the method to nonzero temperature so that one could study how the effects of quantum fluctuations vary with temperature.

\section{ACKNOWLEDGMENTS}

This work was supported in part by the U.S. Department of Energy, Division of High Energy Physics, under Grant No. DE-FG02-91-ER40690. We thank T.-L. Ho for valuable discussions.

\section{APPENDIX A: INTEGRALS}

In this appendix we give analytic expressions for the frequency integrals and the wave-vector integrals that are required to calculate the one-loop quantum corrections to the density and the condensate. Since time-independent sources do not change the energy, the frequency integrals are rather simple. They can be evaluated using contour integration. The specific integrals that are required are

$$
\begin{aligned}
& \int \frac{d \omega}{2 \pi} \frac{1}{\left(\omega^{2}-\epsilon^{2}\right)^{n}} \\
& \quad=i(-1)^{n+1} \frac{(-1) \times 1 \times 3 \cdots(2 n-3)}{2^{n}(n-1) !} \frac{1}{\epsilon^{2 n-1}},
\end{aligned}
$$




$$
\begin{aligned}
& \int \frac{d \omega}{2 \pi} \frac{\omega^{2}}{\left(\omega^{2}-\epsilon^{2}\right)^{n+1}} \\
& \quad=i(-1)^{n+1} \frac{(-1) \times 1 \times 3 \cdots(2 n-3)}{2^{n+1} n !} \frac{1}{\epsilon^{2 n-1}} .
\end{aligned}
$$

Time-independent sources that are inserted into a loop diagram change the wave vector $\mathbf{k}$ of the propagators in the loop. The gradient expansion corresponds to expanding the loop integral in powers of the wave vectors $\mathbf{p}_{i}$ of the sources and of the external lines of the diagram. After averaging over integration angles, the $k$ integrals that are required have the form

$$
I_{m, n} \equiv \int \frac{d^{3} k}{(2 \pi)^{3}} \frac{\left(k^{2}\right)^{m}}{\left(k \sqrt{k^{2}+\Lambda^{2}}\right)^{n}},
$$

where $m$ and $n$ are integers. If $m$ and $n$ satisfy $m-n<-3 / 2$, this integral is ultraviolet convergent. If $2 m-n>-3$, the integral is infrared convergent. If it is both ultraviolet and infrared convergent, its value is

$$
\begin{gathered}
I_{m, n}=\frac{\Gamma(n-m-3 / 2) \Gamma((3-n) / 2+m)}{4 \pi^{2} \Gamma(n / 2)} \Lambda^{3+2 m-2 n}, \\
m+\frac{3}{2}<n<2 m+3 .
\end{gathered}
$$

The ultraviolet-divergent integrals that are required are $I_{m, n}$ for $n-m=-1,0,1$, which have power ultraviolet divergences. A convenient way to regularize the integral is to subtract pure powers of $k$ from the integrand that will remove the ultraviolet divergence and then to add those powers of $k$ back in with an ultraviolet cutoff $k<\Lambda_{\mathrm{UV}}$. The regularized integral is then

$$
\begin{aligned}
I_{m, n} \equiv & \frac{1}{2 \pi^{2}} \int_{0}^{\infty} d k\left[\frac{k^{2+2 m-n}}{\left(k^{2}+\Lambda^{2}\right)^{n / 2}}\right. \\
& \left.-\sum_{i=0}^{m-n+1}\left(-\frac{n}{2}\right) \Lambda^{2 i} k^{2+2 m-2 n-2 i}\right] \\
& +\frac{1}{2 \pi^{2}} \sum_{i=0}^{m-n+1}\left(-\frac{n}{2}\right) \Lambda^{2 i} \int_{0}^{\Lambda_{\mathrm{UV}}} d k k^{2+2 m-2 n-2 i}, \\
& n<m+\frac{3}{2} .
\end{aligned}
$$

The first integral in Eq. (A5) is convergent and is equal to the expression on the right-hand side of Eq. (A4). The only dependence on the ultraviolet cutoff comes from the remaining integrals in Eq. (A5), and they give a polynomial in $\Lambda_{\mathrm{UV}}$. The ultraviolet divergent integrals that arise in our calculation are

$$
I_{n-1, n}=\frac{1}{4 \pi^{2}}\left(2 \Lambda_{\mathrm{UV}}+\frac{\Gamma(-1 / 2) \Gamma((n+1) / 2)}{\Gamma(n / 2)} \Lambda\right),
$$

$$
\begin{gathered}
I_{n, n}=\frac{1}{4 \pi^{2}}\left(\frac{2}{3} \Lambda_{\mathrm{UV}}^{3}-n \Lambda_{\mathrm{UV}} \Lambda^{2}+\frac{\Gamma(-3 / 2) \Gamma((n+3) / 2)}{\Gamma(n / 2)} \Lambda^{3}\right), \\
I_{n+1, n}=\frac{1}{4 \pi^{2}}\left(\frac{2}{5} \Lambda_{\mathrm{UV}}^{5}-\frac{n}{3} \Lambda_{\mathrm{UV}}^{3} \Lambda^{2}+\frac{n(n+2)}{4} \Lambda_{\mathrm{UV}} \Lambda^{4}\right. \\
\left.+\frac{\Gamma(-5 / 2) \Gamma((n+5) / 2)}{\Gamma(n / 2)} \Lambda^{5}\right) .
\end{gathered}
$$

The infrared-divergent integrals that are required are $I_{m, n}$ for $n=2 m+3$, which have logarithmic infrared divergences. The integrals can be regularized by imposing an infrared cutoff $k>\lambda_{\mathrm{IR}}$. In the limit $\lambda_{\mathrm{IR}} \ll \Lambda$, the regularized integral reduces to

$$
\begin{aligned}
I_{m, 2 m+3} \equiv & \frac{1}{2 \pi^{2}} \int_{0}^{\infty} d k\left(\frac{1}{k\left(k^{2}+\Lambda^{2}\right)^{m+3 / 2}}-\frac{\theta(\Lambda-k)}{k \Lambda^{2 m+3}}\right) \\
& +\frac{1}{2 \pi^{2}} \Lambda^{-2 m-3} \log \frac{\Lambda}{\lambda_{\mathrm{IR}}}
\end{aligned}
$$

The specific integrals that are needed in our calculations are

$$
\begin{gathered}
I_{-2,-1}=\frac{1}{4 \pi^{2}}\left[2 \Lambda_{\mathrm{UV}}+\left(\log \frac{4 \Lambda^{2}}{\lambda_{\mathrm{IR}}^{2}}-2\right) \Lambda\right], \\
I_{-1,1}=\frac{1}{4 \pi^{2}}\left(\log \frac{4 \Lambda^{2}}{\lambda_{\mathrm{IR}}^{2}}\right) \frac{1}{\Lambda} \\
I_{0,3}=\frac{1}{4 \pi^{2}}\left(\log \frac{4 \Lambda^{2}}{\lambda_{\mathrm{IR}}^{2}}-2\right) \frac{1}{\Lambda^{3}} .
\end{gathered}
$$

Note that the integral $I_{-2,-1}$ is ultraviolet divergent as well as infrared divergent.

\section{APPENDIX B: EXPLICIT CALCULATION OF A DIAGRAM}

In this appendix we illustrate the calculation of one-loop diagrams that contribute to the quantum corrections to the density by calculating one diagram in detail. We consider the last diagram in Fig. 3, which represents a contribution to the matrix element $\left\langle\xi^{2}\right\rangle_{X, Y}$ involving two insertions of the source $X$.

It is convenient to calculate the diagram in wave-vector space, and then Fourier transform to get the diagram in coordinate space. The diagram involves an integral over the frequency $\omega$ and an integral over the wave vector $\mathbf{k}$ running around the loop. Letting the wave vectors of the two sources be $\mathbf{p}_{1}$ and $\mathbf{p}_{2}$, the expression for the diagram is

$$
\begin{aligned}
\frac{1}{2} \int \frac{d \omega}{2 \pi} \int \frac{d^{3} k}{(2 \pi)^{3}} 2 \frac{i\left(\mathbf{k}+\mathbf{p}_{1}\right)^{2} /(2 m)}{\omega^{2}-\epsilon^{2}\left(\left|\mathbf{k}+\mathbf{p}_{1}\right|\right)} \\
\quad \times \frac{i X\left(\mathbf{p}_{1}\right)}{2 m} \frac{i k^{2} / 2 m}{\omega^{2}-\epsilon^{2}(k)} \frac{i X\left(\mathbf{p}_{2}\right)}{2 m} \frac{i\left(\mathbf{k}-\mathbf{p}_{2}\right)^{2} / 2 m}{\omega^{2}-\epsilon^{2}\left(\left|\mathbf{k}-\mathbf{p}_{2}\right|\right)} .
\end{aligned}
$$

We have written the Feynman rules for each of the propagators and vertices in the loop in the order in which they appear as one goes clockwise around the loop. There is a symmetry 
factor of $1 / 2$ and the factor of 2 inside the integral is the Feynman rule for the operator $\xi^{2}$. There is an implied $+i 0^{+}$ prescription in the denominator of each of the propagators.

The first step in evaluating the diagram is to expand the integrand to second order in powers of the external wave vectors $\mathbf{p}_{1}$ and $\mathbf{p}_{2}$. The expansion of the denominators has the form

$$
\begin{aligned}
& \frac{1}{\omega^{2}-\epsilon^{2}(|\mathbf{k}+\mathbf{p}|)}=\frac{1}{\omega^{2}-\epsilon^{2}(k)} \\
& +\left[\left(\frac{k^{2}}{2 m}+\frac{\epsilon^{2}(k)}{k^{2} / 2 m}\right) \frac{p^{2}+2 \mathbf{p} \cdot \mathbf{k}}{2 m}+\frac{(\mathbf{p} \cdot \mathbf{k})^{2}}{m^{2}}\right] \frac{1}{\left[\omega^{2}-\epsilon^{2}(k)\right]^{2}} \\
& +\left(\frac{k^{2}}{2 m}+\frac{\epsilon^{2}(k)}{k^{2} / 2 m}\right)^{2} \frac{(\mathbf{p} \cdot \mathbf{k})^{2}}{m^{2}\left[\omega^{2}-\epsilon^{2}(k)\right]^{3}} .
\end{aligned}
$$

We can now use the formula (A1) to evaluate the integral over $\omega$. This reduces the diagram to an integral over $\mathbf{k}$. We can average over the angles of $\mathbf{k}$ by making the substitutions $k^{i} k^{j} \rightarrow k^{2} \delta^{i j} / 3$ and $k^{i} \rightarrow 0$. After simplifying the diagram, we find the terms

$$
\begin{aligned}
& \frac{1}{16(2 m)^{2}} X\left(\mathbf{p}_{1}\right) X\left(\mathbf{p}_{2}\right) \int \frac{d^{3} k}{(2 \pi)^{3}}\left\{3 \frac{\left(k^{2} / 2 m\right)^{3}}{\epsilon^{5}(k)}\right. \\
& -\frac{\mathbf{p}_{1} \cdot \mathbf{p}_{2}}{6(2 m)}\left[35 \frac{\left(k^{2} / 2 m\right)^{6}}{\boldsymbol{\epsilon}^{9}(k)}-10 \frac{\left(k^{2} / 2 m\right)^{4}}{\boldsymbol{\epsilon}^{7}(k)}+3 \frac{\left(k^{2} / 2 m\right)^{2}}{\boldsymbol{\epsilon}^{5}(k)}\right] .
\end{aligned}
$$

There are also terms proportional to $\left(p_{1}^{2}+p_{2}^{2}\right) X\left(\mathbf{p}_{1}\right) X\left(\mathbf{p}_{2}\right)$ that we have dropped. They correspond to terms of the form $X \nabla^{2} X$, which first contribute to the density at fourth order in the gradient expansion. Expressing the integrals over $\mathbf{k}$ in Eq. (B3) in terms of the integrals $I_{m, n}$ defined in Appendix A, the expression reduces to

$$
\begin{aligned}
& \frac{3}{16} X\left(\mathbf{p}_{1}\right) X\left(\mathbf{p}_{2}\right) I_{3,5} \\
& -\frac{1}{192}\left(35 I_{6,9}-10 I_{4,7}+3 I_{2,5}\right) \mathbf{p}_{1} \cdot \mathbf{p}_{2} X\left(\mathbf{p}_{1}\right) X\left(\mathbf{p}_{2}\right) .
\end{aligned}
$$

After Fourier transforming to coordinate space, this becomes

$$
\frac{3}{16} I_{3,5} X^{2}(\mathbf{r})+\frac{1}{192}\left(35 I_{6,9}-10 I_{4,7}+3 I_{2,5}\right)(\nabla X)^{2}(\mathbf{r}) \text {. }
$$

From this expression, we can now read off the coefficients $a_{4}$ and $a_{5}$ in the expansion (51) for $\left\langle\xi^{2}\right\rangle_{X, Y}$.

\section{APPENDIX C: COEFFICIENTS}

In this appendix we express the coefficients that appear in the calculation of one-loop quantum corrections to the density in terms of the integrals $I_{m, n}$ that were defined in Appendix A. We first list the coefficients that are used in Secs. IV and $\mathrm{V}$ to calculate the condensate and the density profile using the Cartesian field parametrization. The coefficients in the expansion (51) for $\left\langle\xi^{2}\right\rangle_{X, Y}$ are

$$
\begin{gathered}
a_{0}=\frac{1}{2} I_{1,1}, \\
a_{1}=\frac{1}{4} I_{2,3}, \\
a_{2}=-\frac{1}{4} I_{0,1}, \\
a_{3}=\frac{1}{48}\left(-10 I_{5,7}+13 I_{3,5}-I_{1,3}\right), \\
a_{4}=\frac{3}{16} I_{3,5}, \\
\frac{1}{192}\left(35 I_{6,9}-10 I_{4,7}+3 I_{2,5}\right) .
\end{gathered}
$$

The coefficients in the expansion (52) for $\left\langle\eta^{2}\right\rangle_{X, Y}$ are

$$
\begin{aligned}
& b_{0}=\frac{1}{2} I_{-1,-1}, \\
& b_{1}=-\frac{1}{4} I_{0,1}, \\
& b_{2}=\frac{1}{4} I_{-2,-1},
\end{aligned}
$$

$$
\begin{gathered}
b_{3}=\frac{1}{48}\left(2 I_{3,5}-3 I_{1,3}-I_{-1,1}\right), \\
b_{4}=-\frac{1}{16} I_{1,3},
\end{gathered}
$$

$$
b_{5}=-\frac{5}{192}\left(I_{4,7}+2 I_{2,5}+I_{0,3}\right) \text {. }
$$

We next list the coefficients that are required in Sec. VI to calculate the density profile using the polar field parametrization. The coefficients in the expansion (112) for $\left\langle\xi^{2}\right\rangle_{X, U, S}$ are

$$
\begin{gathered}
c_{0}=\frac{1}{2} I_{1,1}, \\
c_{1}=\frac{1}{4} I_{3,3}, \\
c_{2}=-\frac{1}{4} I_{1,1}, \\
c_{3}=\frac{3}{16} I_{5,5}, \\
c_{4}=-\frac{1}{8} I_{3,3},
\end{gathered}
$$




$$
c_{5}=-\frac{1}{16} I_{1,1}
$$

The coefficients in the expansion (113) for $\left\langle(\nabla \xi)^{2}\right\rangle_{X, U, S}$ are

$$
\begin{gathered}
d_{0}=\frac{1}{2} I_{2,1}, \\
d_{1}=\frac{1}{4} I_{3,3}, \\
d_{2}=\frac{1}{48}\left(-10 I_{7,7}+25 I_{5,5}-9 I_{3,3}\right), \\
d_{3}=\frac{1}{48}\left(2 I_{5,5}-7 I_{3,3}-I_{1,1}\right), \\
d_{4}=\frac{1}{192}\left(35 I_{9,9}-90 I_{7,7}+91 I_{5,5}\right), \\
d_{5}=\frac{1}{96}\left(-5 I_{7,7}+14 I_{5,5}-21 I_{3,3}\right), \\
d_{6}=\frac{1}{192}\left(-5 I_{5,5}+6 I_{3,3}-13 I_{1,1}\right) .
\end{gathered}
$$

The coefficients in the expansion (114) for $\left\langle(\nabla \eta)^{2}\right\rangle_{X, U, S}$ are

$$
e_{0}=\frac{1}{2} I_{0,-1}
$$

$$
e_{1}=-\frac{1}{4} I_{1,1}
$$

$$
e_{2}=\frac{1}{48}\left(2 I_{5,5}-7 I_{3,3}-I_{1,1}\right)
$$

$$
e_{3}=\frac{1}{48}\left(2 I_{3,3}-3 I_{1,1}+7 I_{-1,-1}\right)
$$

$$
e_{4}=\frac{1}{192}\left(-5 I_{7,7}+6 I_{5,5}-13 I_{3,3}\right) \text {, }
$$

$$
e_{5}=\frac{1}{96}\left(3 I_{5,5}-2 I_{3,3}-13 I_{1,1}\right)
$$

$$
e_{6}=\frac{1}{64}\left(I_{3,3}+2 I_{1,1}+9 I_{-1,-1}\right) \text {. }
$$

${ }^{1}$ M. H. Anderson et al., Science 269, 198 (1995).

${ }^{2}$ C. C. Bradley et al., Phys. Rev. Lett. 75, 1687 (1995).

${ }^{3}$ K. B. Davis et al., Phys. Rev. Lett. 75, 3969 (1995).

${ }^{4}$ M. Edwards and K. Burnett, Phys. Rev. A 51, 1382 (1995); M. Edwards et al., ibid. 53, R1950 (1996).

${ }^{5}$ F. Dalfovo and S. Stringari, Phys. Rev. A 53, 2477 (1996).

${ }^{6}$ G. Baym and C. J. Pethick, Phys. Rev. Lett. 76, 6 (1996).

${ }^{7}$ R. J. Dodd, J. Res. Natl. Inst. Stand. Technol. 101, 537 (1996).

${ }^{8}$ K. N. Ilinski and A. Moroz, J. Res. Natl. Inst. Stand. Technol. 101, 567 (1996).

${ }^{9}$ A. L. Fetter, J. Low Temp. Phys. 106, 643 (1997).

${ }^{10}$ F. Dalfovo, L. Pitaevskii, and S. Stringari, J. Res. Natl. Inst. Stand. Technol. 101, 537 (1996); Phys. Rev. A 54, 4213 (1996). ${ }^{11}$ A. L. Fetter and D. L. Feder, cond-mat/9704173(unpublished).

${ }^{12}$ A. Griffin, Phys. Rev. B 53, 9341 (1996).

${ }^{13}$ T. T. Chou, C. N. Yang, and L. H. Yu, cond-mat/9602153 (unpublished); Phys. Rev.B 55, 1179 (1997).

${ }^{14}$ K. Huang, cond-mat/9609033 (unpublished); E. Timmermans, P. Tommasini, and K. Huang, Phys. Rev. A 55, 3645 (1997).

${ }^{15}$ R. Jackiw, in M.A.B. Beg Memorial Volume, edited by A. Ali and P. Hoodbhoy (World Scientific, Singapore, 1991), pp. 25-42.

${ }^{16}$ E. Braaten and A. Nieto, Phys. Rev. B 55, 8090 (1997).

${ }^{17}$ K. Huang and C. N. Yang, Phys. Rev. 105, 767 (1957).

${ }^{18}$ T. D. Lee and C. N. Yang, Phys. Rev. 105, 1119 (1957); T. D. Lee, K. Huang, and C. N. Yang, ibid. 106, 1135 (1957); C. N. Yang, Physica (Amsterdam) 26, S49 (1960).

${ }^{19}$ T. Haugset, H. Haugerud, and F. Ravndal, cond-mat/9706088 (unpublished).

${ }^{20}$ K. Kirsten and D. J. Toms, J. Res. Natl. Inst. Stand. Technol. 101, 471 (1996)

${ }^{21}$ K. N. Ilinski and A. S. Stepanenko, cond-mat/9612117 (unpublished). 